Ideais de anéis de operadores diferenciais 


\title{
Ideais de anéis de operadores diferenciais ${ }^{1}$
}

\author{
Napoleón Caro Tuesta
}

Orientador: Prof. Dr. Daniel Levcovitz

Tese apresentada ao Instituto de Ciências Matemáticas e de Computação - ICMC-USP, como parte dos requisitos para obtenção do título de Doutor em Ciências - Matemática. EXEMPLAR DE DEFESA.

USP - São Carlos

Fevereiro de 2011

\footnotetext{
${ }^{1}$ Este trabalho teve suporte financeiro da CAPES
} 
Ficha catalográfica elaborada pela Biblioteca Prof. Achille Bassi e Seção Técnica de Informática, ICMC/USP, com os dados fornecidos pelo(a) autor(a)

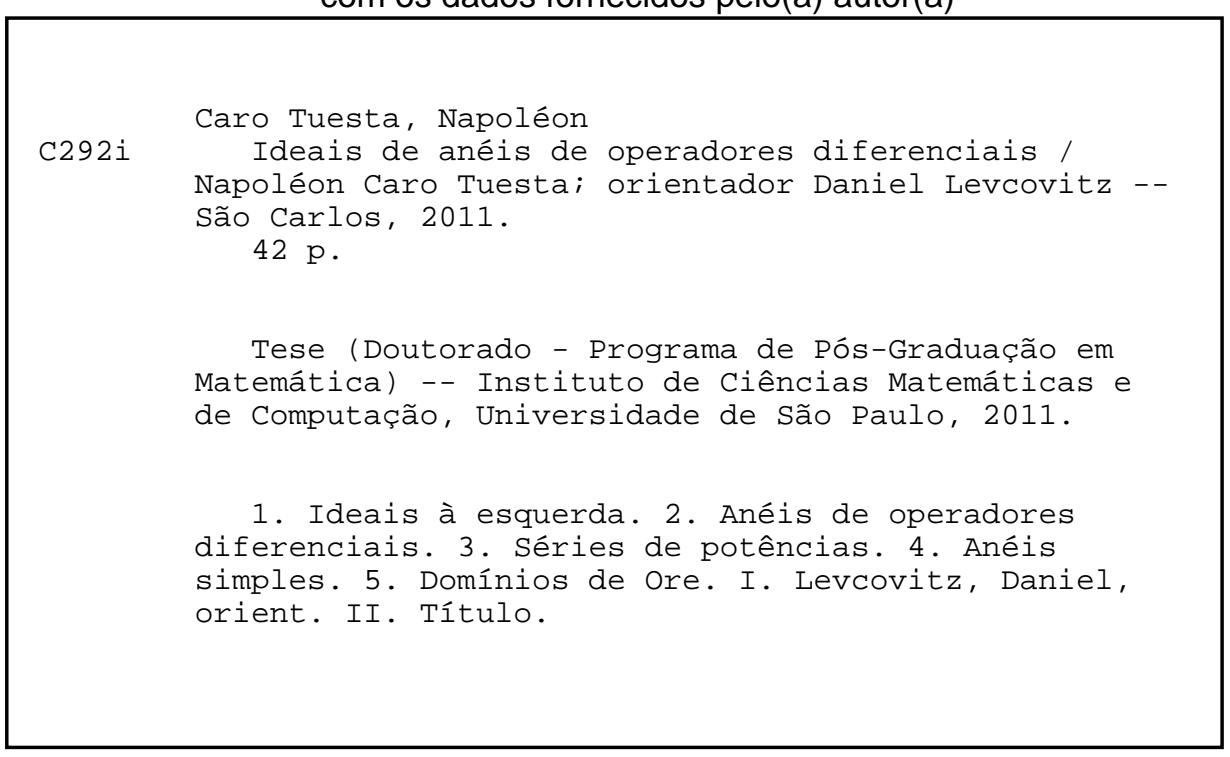


Um matemático que não é também um pouco poeta nunca será um matemático completo. Karl Weierstrass. 
Este trabalho está dedicado a minha esposa Elizabeth, meus pais Napoleón e Aleida e minha irmã Sheila com todo теи $\rho=1-\sin (\theta)$. 


\section{Agradecimentos}

Agradeço aos que mais amo, minha família. Aos meus pais Napoleón e Aleida, a minha esposa Elizabeth, minha irmã Sheila e minha sobrinha querida Gabrielita, pelo infinito amor.

Agradeço especialmente ao Professor Daniel Levcovitz, meu orientador, pela amizade e apoio, pelos conhecimentos ensinados e por apresentar-me o fascinante mundo da álgebra não comutativa.

Agradeço aos professores do ICMC-USP, com os quais aprendi muito. De maneira especial agradeço a Marcelo Saia, Víctor Hugo, Maria Aparecida, Raimundo Nonato e Eduardo Tengan. Agradeço também ao professor Roberto Bedregal da UFPB, pela amizade e por nossas longas conversas da matemática e da vida em geral.

Agradeço a meus colegas e amigos do ICMC-USP, de forma especial a Walter Huaraca, Lizandro Sánchez, José Bravo, Nancy Chachapoyas, Manuel Zuloeta, Luis Espinoza , Chistian Surco, Pedro (Ceará) e Renato Dias. Obrigado pelo apoio, pelas longas conversas, pelas horas de estudo, pelos dias de futebol e por todos os momentos inesquecíveis vividos.

Agradeço a CAPES, Coordenação de Aperfeiçoamento de Pessoal de Nível Superior, pelo apoio financeiro.

\section{Muito Obrigado!}




\section{Resumo}

Em [12] J.T. Stafford demonstrou que todo ideal à esquerda ou à direita da álgebra de Weyl $A_{n}(K)=K\left[x_{1}, \ldots, x_{n}\right]\left\langle\partial_{1}, \ldots, \partial_{n}\right\rangle$ ( $K$ um corpo de característica zero) é gerado por dois elementos. Consideremos o anel $D_{n}:=K\left[\left[x_{1}, \ldots, x_{n}\right]\right]\left\langle\partial_{1}, \ldots, \partial_{n}\right\rangle$ de operadores diferenciais sobre o anel de séries de potências formais $K\left[\left[x_{1}, \ldots, x_{n}\right]\right]$. Uma pergunta natural é se todo ideal à esquerda ou à direita de $D_{n}(K)$ pode ser gerado por dois elementos.

Neste trabalho provaremos que todo ideal à esquerda ou à direita do anel $E_{n}(K):=K\left(\left(x_{1}, \ldots, x_{n}\right)\right)\left\langle\partial_{1}, \ldots, \partial_{n}\right\rangle$ de operadores diferenciais sobre o corpo das séries de Laurent $K\left(\left(x_{1}, \ldots, x_{n}\right)\right)$ é gerado por dois elementos. Nós provaremos também que todo ideal à esquerda ou à direita do anel $S_{n-1}(K):=K\left(\left(x_{1}, \ldots, x_{n-1}\right)\right)\left[\left[x_{n}\right]\right]\left\langle\partial_{1}, \ldots, \partial_{n}\right\rangle$ é gerado por dois elementos e como corolário obtemos uma demonstração que todo ideal à esquerda ou à direita do anel $D_{1}(K)$ é gerado por dois elementos. Isto está de acordo com a conjectura que diz que todo ideal à esquerda ou à direita de um anel (não comutativo) Noetheriano simples é gerado por dois elementos. 


\section{Abstract}

In [12] J.T. Stafford proved that every left or right ideal of the Weyl algebra $A_{n}(K)=K\left[x_{1}, \ldots, x_{n}\right]\left\langle\partial_{1}, \ldots, \partial_{n}\right\rangle$ ( $K$ a field of characteristic zero) is generated by two elements. Consider the ring $D_{n}:=K\left[\left[x_{1}, \ldots, x_{n}\right]\right]\left\langle\partial_{1}, \ldots, \partial_{n}\right\rangle$ of differential operators over the ring of formal power series $K\left[\left[x_{1}, \ldots, x_{n}\right]\right]$. A natural question is that if every left or right ideal of $D_{n}(K)$ can be generated by two elements.

In this work we will prove that every left or right ideal of the ring $E_{n}(K):=K\left(\left(x_{1}, \ldots, x_{n}\right)\right)\left\langle\partial_{1}, \ldots, \partial_{n}\right\rangle$ of differential operators over the field of formal Laurent series $K\left(\left(x_{1}, \ldots, x_{n}\right)\right)$ is generated by two elements. We will prove also that every left or right ideal of the ring $S_{n-1}(K):=K\left(\left(x_{1}, \ldots, x_{n-1}\right)\right)\left[\left[x_{n}\right]\right]\left\langle\partial_{1}, \ldots, \partial_{n}\right\rangle$ is generated by two elements and as a corollary we obtain a proof of that every left or right ideal of the ring $D_{1}(K)$ is generated by two elements. This is in accordance with the conjecture that says that in a (noncommutative) Noetherian simple ring, every left or right ideal is generated by two elements. 


\section{Sumário}

Introdução 1

1 Preliminares 3

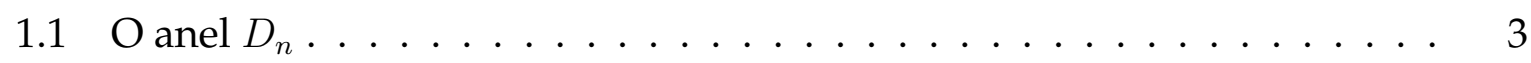

1.2 Domínios de Ore $\ldots \ldots \ldots \ldots \ldots$

2 O Caso $n=1, D_{1}(K)=K[[x]]\left\langle\partial_{x}\right\rangle$

2.1 Bases de Divisão . . . . . . . . . . . . . . . . . . . . . . . . . . . 14

2.2 Os ideais á esquerda de $D_{1}(K)$ são dois gerados. . . . . . . . . . . . . . . 17

$3 \quad$ Módulos Livres sobre $S=T((x))\left\langle\partial_{x}\right\rangle$ e sobre $D=F[[x]]\left\langle\partial_{x}\right\rangle$

$3.1 \quad$ Propriedades dos anéis $S$ e $D \ldots \ldots$. . . . . . . . . . . 19

$3.1 .1 \quad \mathrm{O}$ anel $S=T((x))\left\langle\partial_{x}\right\rangle \ldots \ldots \ldots \ldots$. . . . . . . . . . 19

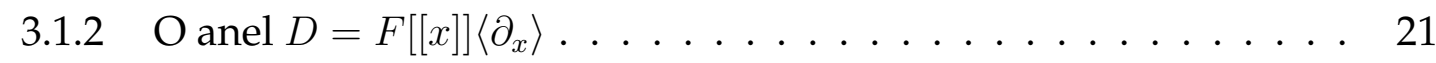

3.2 Módulos livres . . . . . . . . . . . . . . . . . . . . . . . . . 22

$\begin{array}{lll}4 & \text { Resultados Principais } & 28\end{array}$

$4.1 \quad$ Os anéis $B_{r}(K) \ldots \ldots \ldots \ldots \ldots \ldots \ldots \ldots$

$4.2 \quad \mathrm{O}$ anel $E_{n}(K)=K\left(\left(x_{1}, \ldots, x_{n}\right)\right)\left\langle\partial_{1}, \ldots, \partial_{n}\right\rangle \ldots \ldots \ldots \ldots$

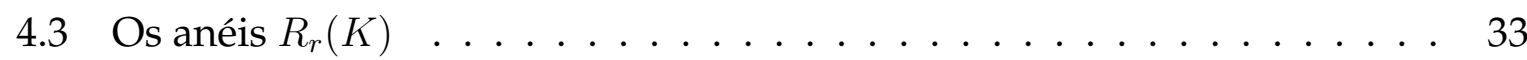

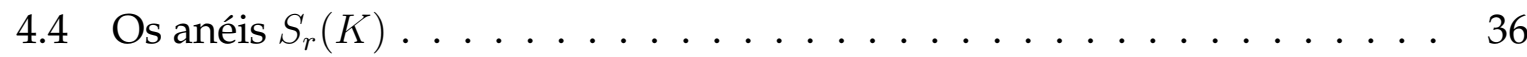

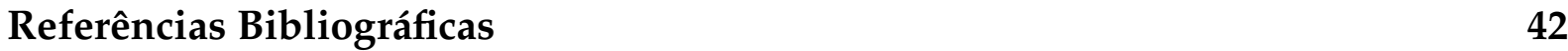





\section{Introdução}

Seja $K$ um corpo de característica zero e seja $A_{n}(K)=K\left[x_{1}, \ldots, x_{n}\right]\left\langle\partial_{1}, \ldots, \partial_{n}\right\rangle$, $\left(\partial_{i}:=\partial_{x_{i}}\right)$, a $n$-ésima álgebra de Weyl sobre $K$. Esta $K$-álgebra é associativa e gerada pelos $x$ 's e pelas $\partial$ 's satisfazendo as relações:

$$
\left[x_{i}, x_{j}\right]=0=\left[\partial_{i}, \partial_{j}\right], \quad\left[\partial_{i}, x_{j}\right]=\delta_{i j}, \quad \text { para todo } i, j=1, \ldots, n .
$$

Ela pode ser pensada como o anel de operadores diferenciais com coeficientes polinomiais ou ainda como o anel de operadores diferencias da variedade algébrica afim $\mathbb{A}_{K}^{n}$.

Sabemos que $A_{n}(K)$ é uma álgebra não comutativa simples (sem ideais bilaterias não triviais), sem divisores de zero e Noetheriana.

Em 1978, J.T. Stafford demonstrou um importante resultado sobre a estrutura dos ideais de $A_{n}(K)$. Ele provou que todo ideal à esquerda (ou à direita) de $A_{n}(K)$ pode ser gerado por dois elementos. Este fato aparece no artigo original de Stafford [12]. Uma simplificação da prova encontra-se no Capítulo 1 do livro de Björk [1].

Uma questão que surge naturalmente é se esse resultado ainda é válido para outros anéis Noetherianos simples. Dizemos que um anel é $n$-gerado se todo ideal à esquerda ou à direita pode ser gerado por $n$ elementos. Assim, a álgebra de Weyl $A_{n}(K)$ é 2-gerado. Um outro exemplo são os anéis de operadores diferenciais $D(X)$ sobre uma variedade algébrica afim suave. Nesse caso, Coutinho e Holland [4] provaram que $D(X)$ é 3-gerado, embora acredita-se que ele seja de fato 2-gerado. Na verdade, uma conjectura importante nessa área é:

Conjectura: Todo anel Noetheriano simples é 2-gerado.

Essa conjectura está aberta em geral.

Nessa tese vamos discutir o caso do anel $D_{n}(K)=K\left[\left[x_{1}, \ldots, x_{n}\right]\right]\left\langle\partial_{1}, \ldots, \partial_{n}\right\rangle$, o anel de operadores diferenciais do anel de séries de potências $K\left[\left[x_{1}, \ldots, x_{n}\right]\right]$. Mais especificamente nos perguntamos se $D_{n}(K)$ é 2-gerado. Essa pergunta enquadra-se na conjec- 
tura acima, dado que o anel $D_{n}(K)$ é um domínio Noetheriano simples. O caso $n=1$ é verdadeiro e está demonstrado em [6]. Assim $D_{1}(K)$ é 2- gerado.

Embora não demonstramos nessa tese que $D_{n}(K)$ é 2-gerado, mostraremos que a conjectura é verdadeira para o anel $E_{n}(K)=K\left(\left(x_{1}, \ldots, x_{n}\right)\right)\left\langle\partial_{1}, \ldots, \partial_{n}\right\rangle$, o anel de operadores diferenciais de $K\left(\left(x_{1}, \ldots, x_{n}\right)\right)$, que é o corpo de frações de $K\left[\left[x_{1}, . ., x_{n}\right]\right]$ (Teorema 4.8, pág 33). Para isto, seguiremos as técnicas do Stafford e Björk e as adaptaremos ao nosso caso. Também mostraremos que o anel $S_{n-1}(K)=K\left(\left(x_{1}, \ldots, x_{n-1}\right)\right)\left[\left[x_{n}\right]\right]\left\langle\partial_{1}, \ldots, \partial_{n}\right\rangle$ é 2-gerado (Teorema 4.13, pág 38). Como consequência obtemos uma nova prova que $D_{1}(K)$ é 2-gerado.

Este trabalho está divido em quatro capítulos. No primeiro, desenvolvemos os preliminares necessários para a leitura da tese. Introduzimos o anel $D_{n}(K)$ e mostramos suas propriedades fundamentais. Faremos também uma introdução aos domínios de Ore e seus anéis de frações.

No capítulo 2 mostraremos que $D_{1}(K)$ é 2-gerado, seguindo [6].

O capítulo 3 destina-se a estudar módulos livres sobre os anéis $S=T((x))\left\langle\partial_{x}\right\rangle$ e $D_{1}(F)=F[[x]]\left\langle\partial_{x}\right\rangle$, onde $T$ é um anel de divisão de característica zero e $F$ é um corpo também de característica zero. Neste capítulo desenvolveremos resultados técnicos sobre módulos livres que serão usados no último capítulo.

O capítulo 4 contém os teoremas principais dessa tese: o Teorema 4.8, que mostra que $E_{n}(K)$ é 2-gerado e o Teorema 4.13 que mostra que $S_{n-1}(K)$ é 2-gerado.

Uma palavra final sobre a diferença entre o caso da álgebra de Weyl $A_{n}(K)$ e o caso do anel $D_{n}(K)$. A dificuldade em se estender os métodos da demonstração do caso da álgebra de Weyl (que é o anel de operadores diferenciais do anel de polinômios), para o caso do anel $D_{n}(K)$ (que é o anel de operadores diferenciais do anel de séries de potências), reside na quebra de simetria entre as variáveis $x^{\prime} s$ e $\partial$ 's. Enquanto que na álgebra de Weyl elas são simétricas, como mostra as relações de definição, no caso de $D_{n}(K)$ essa simetria é quebrada. De fato, no anel $D_{n}(K)=K\left[\left[x_{1}, \ldots, x_{n}\right]\right]\left\langle\partial_{1}, \ldots, \partial_{n}\right\rangle$ admita-se somas infinitas (séries) nas variáveis $x$ 's enquanto somente somas finitas aparecem nas variáveis $\partial$ ' $s$. É essa quebra de simetria que torna o caso $D_{n}(K)$ interessante. 


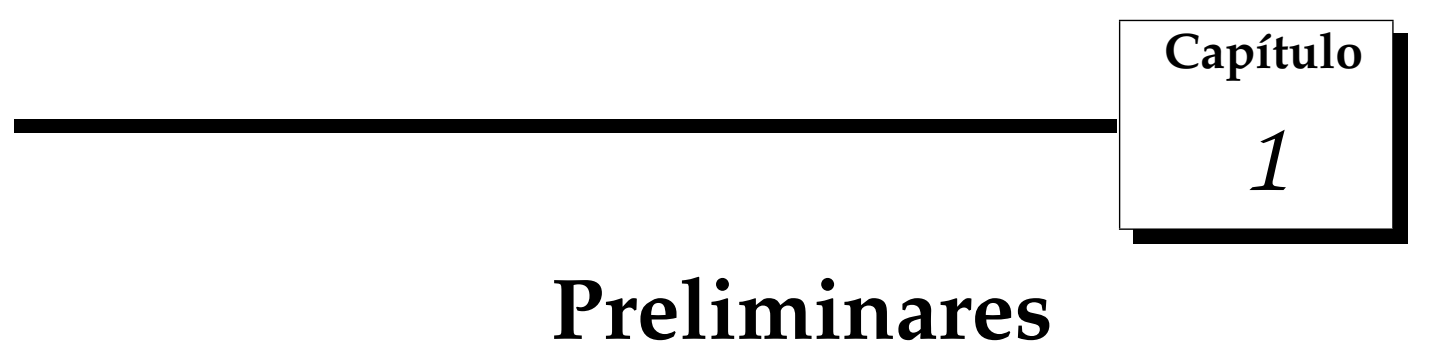

Neste capítulo estudaremos o anel $D_{n}(K)=K\left[\left[x_{1}, \ldots, x_{n}\right]\right]\left\langle\partial_{1}, \ldots, \partial_{n}\right\rangle$, o anel de operadores diferenciais de $K\left[\left[x_{1}, \ldots, x_{n}\right]\right]$, assim como os domínios de Ore. Na primeira seção definiremos $D_{n}(K)$ e estudaremos suas principais propriedades. Na segunda parte estudaremos os domínios de Ore, seus anéis de frações e finalmente mostraremos que $D_{n}(K)$ é um domínio de Ore.

\subsection{Onel $D_{n}$}

Seja $K$ um corpo de característica zero, $n$ um inteiro positivo e $K\left[\left[x_{1}, \ldots, x_{n}\right]\right]$ o anel de séries de potências formais em $n$ indeterminadas com coeficientes em $K$. Sobre $K\left[\left[x_{1}, \ldots, x_{n}\right]\right]$ temos as derivações usuais $\partial_{1}=\partial / \partial x_{1}, \ldots, \partial_{n}=\partial / \partial x_{n}$.

$\mathrm{O}$ operador $K$-linear $\partial_{i}$ que aplica uma série de potência $p$ em $\partial p / \partial x_{i}$ é chamada derivada parcial com respeito a $x_{i}$. É fácil ver que $\partial_{1}, \ldots, \partial_{n}$ são operadores $K$-lineares que comutam dois a dois.

Se $p$ é um elemento de $K\left[\left[x_{1}, \ldots, x_{n}\right]\right]$, então $p$ define um $K$-operador linear sobre $K\left[\left[x_{1}, \ldots, x_{n}\right]\right]$ que leva uma serie $q$ no produto $p q$. Tal operador é chamado operador produto $\mathbf{p}$. Notemos que os operadores produto comutam dois a dois.

Definição 1.1. O subanel dos $K$-operadores lineares de $K\left[\left[x_{1}, \ldots, x_{n}\right]\right]$ que é gerado pelas derivadas parciais $\partial_{1}, \ldots, \partial_{n}$ e os operadores produto definidos pelas series em $K\left[\left[x_{1}, \ldots, x_{n}\right]\right]$ é chamado anel de operadores diferenciais $K$-lineares sobre $K\left[\left[x_{1}, \ldots, x_{n}\right]\right]$, ou simplesmente $o$ anel de operadores diferenciais de $K\left[\left[x_{1}, \ldots, x_{n}\right]\right]$.

Denotaremos este anel por $D_{n}(K)=K\left[\left[x_{1}, \ldots, x_{n}\right]\right]\left\langle\partial_{1}, \ldots, \partial_{n}\right\rangle$. Por definição $D_{n}(K)$ é uma $K$-subálgebra de $\operatorname{End}_{K}\left(K\left[\left[x_{1}, \ldots, x_{n}\right]\right]\right)$. 
Seja $p$ um elemento de $K\left[\left[x_{1}, \ldots, x_{n}\right]\right]$. Em $D_{n}(K)$ temos a seguinte regra de comutação entre $\partial_{i}$ e o operador produto $p$ :

$$
\left[\partial_{i}, p\right]=\partial p / \partial_{x_{i}}
$$

De fato, por definição, $\left[\partial_{i}, p\right]:=\partial_{i} p-p \partial_{i}$. Portanto, se $q$ é um elemento arbitrário de $K\left[\left[x_{1}, \ldots, x_{n}\right]\right]$, temos que

$$
\left[\partial_{i}, p\right] \cdot q=\partial p q / \partial_{x_{i}}-p \partial q / \partial_{x_{i}}=\partial p / \partial_{x_{i}} \cdot q
$$

Em particular temos as seguintes regras de comutação no anel $D_{n}(K)$ :

$$
\left[\partial_{i}, x_{j}\right]=\delta_{i j}, \quad\left[\partial_{i}, \partial_{j}\right]=0, \quad\left[x_{i}, x_{j}\right]=0, \quad 1 \leq i, j \leq n
$$

Um multi-índice $\alpha$ é um elemento de $\mathbb{N}^{n}$, digamos $\alpha=\left(\alpha_{1}, \ldots, \alpha_{n}\right)$. O comprimento de $\alpha$ é definido como $|\alpha|:=\alpha_{1}+\cdots+\alpha_{n} \in \mathbb{N}$. Usaremos a notação $\partial^{\alpha}$ para denotar o operador $\partial_{1}^{\alpha_{1}} \cdots \partial_{n}^{\alpha_{n}}$

Proposição 1.2. Todo elemento em $D_{n}(K)$ pode ser escrito de maneira única como uma soma finita da forma $\sum p_{\alpha} \partial^{\alpha}$, onde $p_{\alpha}$ é um elemento de $K\left[\left[x_{1}, \ldots, x_{n}\right]\right]$.

Prova: Se $p \in K\left[\left[x_{1}, \ldots, x_{n}\right]\right]$ é considerado como um elemento de $D_{n}(K)$, então para cada $1 \leq i \leq n, \partial_{i} p=p \partial_{i}+\partial_{i}(p)$, onde $\partial_{i}(p)=\partial p / \partial x_{i}$ é novamente considerado como um elemento de $K\left[\left[x_{1}, \ldots, x_{n}\right]\right]$. Tais equações permitem mover as $\partial$-variáveis do lado esquerdo para o lado direito e portanto segue-se que todo elemento em $D_{n}(K)$ pode ser escrito como uma soma finita da forma $\sum p_{\alpha} \partial^{\alpha}$.

Agora mostremos que tal escrita é única. Para isto devemos mostrar que se algum $p_{\alpha} \neq 0$, então $D=\sum p_{\alpha} \partial^{\alpha}$ é um elemento diferente de zero em $D_{n}(K)$; mas $D$ é um operador linear de $K\left[\left[x_{1}, \ldots, x_{n}\right]\right], \operatorname{logo} D \neq 0$ se e somente se existe uma série $f$ tal que $D(f) \neq 0$.

Com efeito, seja $\sigma=\left(\sigma_{1}, \ldots, \sigma_{n}\right)$ um multi-índice tal que $p_{\alpha}=0$ para todo multi-índice $\alpha \operatorname{com}|\alpha|<|\sigma|$ e seja $f:=x_{1}^{\sigma_{1}} \cdots x_{n}^{\sigma_{n}} \in K\left[\left[x_{1}, \ldots, x_{n}\right]\right]$. Um cálculo simples mostra que $D(f)=\sigma_{1} ! \cdots \sigma_{n} ! p_{\sigma} \neq 0$ e portanto $D \neq 0$.

Corolário 1.3. O anel $D_{n}(K)$ é um domínio.

Prova: Suponhamos que $D$ e $E$ são elementos diferentes de zero de $D_{n}(K)$. Então, pela proposição anterior, podemos escrever $D$ e $E$ de maneira única como uma soma finita da forma $D=\sum_{\alpha} p_{\alpha} \partial^{\alpha}, E=\sum_{\beta} p_{\beta} \partial^{\beta}$, onde $p_{\alpha}, p_{\beta} \in K\left[\left[x_{1}, \ldots, x_{n}\right]\right]$. Se $\gamma$ e $\lambda$ são 
multi-índices tais que $|\gamma|=\max \left\{|\alpha|, p_{\alpha} \neq 0\right\}$ e $|\lambda|=\max \left\{|\beta|, p_{\beta} \neq 0\right\}$, então $|\gamma|>$ 0 e $|\lambda|>0$, pois $D \neq 0$ e $E \neq 0$. Mais ainda, se escrevemos $D E \in D_{n}(K)$ como uma soma finita da forma $D E=\sum_{\theta} p_{\theta} \partial^{\theta}$, onde $p_{\theta} \in K\left[\left[x_{1}, \ldots, x_{n}\right]\right]$, então $|\gamma|+|\lambda|=$ $\max \left\{|\theta|, p_{\theta} \neq 0\right\}$. Isto implica que $D E \neq 0$ e portanto, $D_{n}(K)$ é um domínio.

Proposição 1.4. O anel $D_{n}(K)$ é simples. Ou seja, se I é um ideal bilateral de $D_{n}(K)$, então $I=\{0\}$ ou $I=D_{n}(K)$.

Prova: Seja $I$ um ideal bilateral de $D_{n}(K)$ diferente do ideal zero. Provaremos que $I=D_{n}(K)$. Com efeito, seja $D \in I$ tal que $D \neq 0$. Escrevemos

$$
D=D_{0}+D_{1} \partial_{n}+\cdots+D_{k} \partial_{n}^{k}
$$

onde $k$ é um inteiro não negativo, $D_{0}, D_{1}, \ldots, D_{k} \in K\left[\left[x_{1}, \ldots, x_{n}\right]\right]\left\langle\partial_{1}, \ldots, \partial_{n-1}\right\rangle$ e $D_{k} \neq 0$. Aqui $K\left[\left[x_{1}, \ldots, x_{n}\right]\right]\left\langle\partial_{1}, \ldots, \partial_{n-1}\right\rangle$ representa o subanel de $D_{n}$ gerado pelos operadores produto $p$ de $K\left[\left[x_{1}, \ldots, x_{n}\right]\right]$ e as derivações $\partial_{1}, \ldots, \partial_{n-1}$.

Se $k \geq 1$, usamos as equações $\left[\partial_{n}^{j}, x_{n}\right]=\partial_{n}^{j} x_{n}-x_{n} \partial_{n}^{j}=j \partial_{n}^{j-1}$ e obtemos que

$$
\left[D, x_{n}\right]=D x_{n}-x_{n} D=D_{1}+2 D_{2} \partial_{n}+\cdots k D_{k} \partial_{n}^{k-1}
$$

Como $I$ é um ideal bilateral, o elemento $D_{1}:=D x_{n}-x_{n} D \in I$. Se $k \geq 2$ procedemos como acima e obtemos $D_{2}:=\left[D_{1}, x_{n}\right]=D_{1} x_{n}-x_{n} D_{1} \in I$ e depois de $k$ etapas vemos que $I$ contém o elemento diferente de zero $k ! D_{k}$ e portanto $I$ contém $D_{k}$.

Seja $E=D_{k}$. Como $E$ é um elemento diferente de zero de $K\left[\left[x_{1}, \ldots, x_{n}\right]\right]\left\langle\partial_{1}, \ldots, \partial_{n-1}\right\rangle$ podemos escrever:

$$
E=E_{0}+E_{1} \partial_{n-1}+\cdots+E_{l} \partial_{n-1}^{l}
$$

onde $l$ é um inteiro não negativo, $E_{0}, E_{1}, \ldots, E_{l} \in K\left[\left[x_{1}, \ldots, x_{n}\right]\right]\left\langle\partial_{1}, \ldots, \partial_{n-2}\right\rangle$ e $E_{l} \neq 0$. Então se procedemos como acima temos que $I$ contém $E_{l}$, que é um elemento diferente de zero de $K\left[\left[x_{1}, \ldots, x_{n}\right]\right]\left\langle\partial_{1}, \ldots, \partial_{n-2}\right\rangle$. Continuando assim é claro que $I$ contém alguma série diferente de zero $p \in K\left[\left[x_{1}, \ldots, x_{n}\right]\right]$. Se $p$ é uma unidade, então $1=p^{-1} p \in I$ e por tanto $I=D_{n}(K)$. No caso que $p$ não é uma unidade, e depois de uma mudança linear nas variáveis $x_{1}, \ldots, x_{n}$ que por sua vez induz um isomorfismo sobre $D_{n}(K)$, podemos supor que $p\left(0, \ldots, 0, x_{n}\right) \neq 0$. Então, pelo Teorema de Preparação de Weierstrass, podemos escrever

$$
p=u\left(p_{0}+p_{1} x_{n}+\cdots+p_{t-1} x_{n}^{t-1}+x_{n}^{t}\right),
$$

onde $t$ é algum inteiro positivo, $u$ é uma unidade de $K\left[\left[x_{1}, \ldots, x_{n}\right]\right]$ e $p_{j} \in K\left[\left[x_{1}, \ldots, x_{n-1}\right]\right]$ 
para cada $0 \leq j \leq t-1$. Então

$$
q=u^{-1} u=p_{0}+p_{1} x_{n}+\cdots+p_{t-1} x_{n}^{t-1}+x_{n}^{t} \in I .
$$

Como $I$ é um ideal bilateral temos que o elemento

$$
q_{1}:=\left[\partial_{n}, q\right]=\partial q / \partial_{x_{n}}=p_{1}+\cdots+(t-1) p_{t-1} x_{n}^{t-2}+t x_{n}^{t-1} \in I .
$$

Novamente, o elemento

$$
q_{2}:=\left[\partial_{n}, q_{1}\right]=\partial q_{1} / \partial_{x_{n}}=2 p_{2}+\cdots+(t-1)(t-2) x_{n}^{t-3}+t(t-1) x_{n}^{t-2} \in I .
$$

Continuando neste assim, é claro que depois de $t$ etapas, o elemento

$$
q_{t}:=\left[\partial_{n}, q_{t-1}\right]=t ! \in I, \text { o que implica que } I=D_{n}(K)
$$

Definição 1.5. Seja $R$ uma $K$-álgebra. Uma família $\left\{F_{i}\right\}_{i \geq 0}$ de $K$-espaços vetoriais é uma filtração ascendente de $R$ se:

1. $F_{0} \subset F_{1} \subset F_{2} \subset \cdots \subset R$,

2. $R=\bigsqcup_{i \geq 0} F_{i}$,

3. $F_{i} F_{j} \subset F_{i+j}$, para todo $i, j \geq 0$.

Por exemplo, a família $F_{i}=\left\{Q \in D_{n}(K): Q=\sum_{|\alpha| \leq i} q_{\alpha} \partial^{\alpha}\right\}, i \geq 0$ dos operadores diferenciais de ordem $\leq i$ é uma filtração ascendente do anel $D_{n}(K)$.

Suponhamos que $F=\left\{F_{i}\right\}_{i \geq 0}$ é uma filtração de uma $K$-álgebra $R$. Vamos construir uma $K$ álgebra graduada associada a esta filtração da seguinte maneira: Para cada $i \geq 0$, seja o $K$-espaço quociente $F(i)=F_{i} / F_{i-1}$, onde $F_{-1}:=0$. Consideremos a soma direta de eles:

$$
F(0) \oplus F(1) \oplus F(2) \oplus \cdots,
$$

este é um $K$-espaço vetorial que denotaremos por $g r^{F}(R)$. Sobre $g r^{F}(R)$ definimos um produto como segue. Consideremos primeiro dois elementos homogêneos em $\operatorname{gr}^{F}(R)$, digamos $f \in F(i)$ e $g \in F(j)$. Escolhemos $D \in F_{i}$ tal que sua imagem $\bar{D}$ em $F(i)$ é $f$ e escolhemos $E \in F_{j}$ tal que $\bar{E}=g$. Agora $D E$ está em $F_{i+j}$. Definimos então o produto $f g$ como a imagem de $D E$ no espaço quociente $F(i+j)$. 
De fato, temos que verificar que este produto está bem definido, ou seja que o elemento $f g$ em $F(i+j)$ não depende das escolhas de $D$ e $E$. Para isto, notemos que se $D_{1}$ é outro elemento tal que $\bar{D}_{1}=f$ em $F(i)$, então a equação $\bar{D}=\bar{D}_{1}$ em $F(i)$ implica que $D-D_{1} \in F_{i-1}$ e portanto $D E-D_{1} E \in F_{i+j-1}$, logo $\bar{D} \bar{E}=\bar{D}_{1} \bar{E}$ em $F(i+j)$. Analogamente podemos provar que $f g$ não depende da escolha de $E$.

Agora temos definido o produto entre elementos homogêneos em $g r^{F}(R)$. Usando o fato que todo elemento de $g r^{F}(R)$ pode ser escrito de maneira única como uma soma finita de elementos homogêneos, podemos definir o produto entre elementos arbitrários de $g r^{F}(R)$.

A $K$-álgebra $g r^{F}(R)$ construída acima é chamada álgebra graduada associada a filtração ascendente $F$ do anel $R$

Agora consideremos a filtração ascendente $F_{i}=\left\{Q \in D_{n}(K): Q=\sum_{|\alpha| \leq i} q_{\alpha} \partial^{\alpha}\right\}$ do anel $D_{n}(K)$. Notemos que $F_{0}=K\left[\left[x_{1}, \ldots, x_{n}\right]\right]$. O anel associado é um anel de polinômios como mostra a seguinte:

Proposição 1.6. O anel $\operatorname{gr}^{F}\left(D_{n}(K)\right)$ é isomorfo a $K\left[\left[y_{1}, \ldots, y_{n}\right]\right]\left[z_{1}, \ldots, z_{n}\right]$, o anel de polinômios em $n$ indeterminadas $z_{1}, \ldots, z_{n}$ com coeficientes no anel de séries de potência $K\left[\left[y_{1}, \ldots, y_{n}\right]\right]$.

\section{Prova:}

Para $i \geq 1$, seja $\sigma_{i}: F_{i} \rightarrow F(i)=F_{i} / F_{i-1}$ a aplicação quociente. Notemos que ela é também $K\left[\left[x_{1}, \ldots, x_{n}\right]\right]$-linear.

Seja agora $a$ um elemento de $\operatorname{grad}^{F}\left(D_{n}(K)\right)$, então a pode ser escrito de maneira única como uma soma finita

$$
a=a_{0}+\left(a_{1}+F_{0}\right)+\cdots+\left(a_{k}+F_{k}\right)=a_{0}+\sigma_{1}\left(a_{1}\right)+\cdots+\sigma_{k}\left(a_{k}\right)
$$

para alguns $a_{i} \in F_{i}, 0 \leq i \leq k$.

Logo $a_{0}=p(x) \in K\left[\left[x_{1}, \ldots, x_{n}\right]\right]$ e para $1 \leq i \leq k, a_{i}=\sum_{|\alpha| \leq i} q_{\alpha, i}(x) \partial^{\alpha}$. Por tanto

$\sigma_{i}\left(a_{i}\right)=\sigma_{i}\left(\sum_{|\alpha| \leq i} q_{\alpha, i}(x) \partial^{\alpha}\right)=\sigma_{i}\left(\sum_{|\alpha|=i} q_{\alpha, i}(x) \partial^{\alpha}\right)=\sum_{|\alpha|=i} q_{\alpha, i}(x) \sigma_{i}\left(\partial^{\alpha}\right)=\sum_{|\alpha|=i} q_{\alpha, i}(x) \sigma_{1}\left(\partial_{1}\right)^{\alpha_{1}} \cdots \sigma_{1}\left(\partial_{n}\right)^{\alpha_{n}}$

então

$$
a=p(x)+\sum_{i=1}^{k} \sum_{|\alpha|=i} q_{\alpha, i}(x) \sigma_{1}\left(\partial_{1}\right)^{\alpha_{1}} \cdots \sigma_{1}\left(\partial_{n}\right)^{\alpha_{n}}
$$

Isto implica que o anel graduado associado $\operatorname{gr}^{F}\left(D_{n}(K)\right)$ é gerado sobre $K\left[\left[x_{1}, \ldots, x_{n}\right]\right]$ pelos elementos $\sigma_{1}\left(\partial_{1}\right), \ldots, \sigma_{1}\left(\partial_{n}\right)$ de $F(1)$

Por outro lado o anel $\operatorname{grad}^{F}\left(D_{n}(K)\right)$ é comutativo. Com efeito, se $a \in F_{i}$ e $b \in F_{j}$, 
então $a b-b a \in F_{i+j-1}$ e por tanto $\sigma_{i+j}(a) \sigma_{i+j}(b)=\sigma_{i+j}(b) \sigma_{i+j}(a)$ para todos os pares de elementos homogêneos em $g r^{F}\left(D_{n}(K)\right)$, o que implica que o anel $g r^{F}\left(D_{n}(K)\right)$ é comutativo.

As etapas anteriores nos permitem definir um homomorfismo sobrejetivo de anéis

$$
\phi: K\left[\left[y_{1}, \ldots, y_{n}\right]\right]\left[z_{1}, \ldots, z_{n}\right] \rightarrow \operatorname{gr}\left(D_{n}(K)\right)
$$

onde

$$
\phi\left(\sum_{\alpha} q_{\alpha}(y) z^{\alpha}\right)=\sum_{\alpha} q_{\alpha}(x) \sigma_{1}\left(\partial_{1}\right)^{\alpha_{n}} \cdots \sigma_{n}\left(\partial_{n}\right)^{\alpha_{n}} .
$$

Mais ainda, ele é injetivo. De fato, se $\sum_{\alpha} q_{\alpha}(y) z^{\alpha}$ é um elemento de $K\left[\left[y_{1}, \ldots, y_{n}\right]\right]\left[z_{1}, \ldots, z_{n}\right]$ tal que $\phi\left(\sum_{\alpha} q_{\alpha}(y) z^{\alpha}\right)=0$, então temos que

$$
\sum_{\alpha} q_{\alpha}(x) \sigma_{1}\left(\partial_{1}\right)^{\alpha_{n}} \cdots \sigma_{n}\left(\partial_{n}\right)^{\alpha_{n}}=q_{0}(x)+\sum_{|\alpha| \geq 1} \sigma_{|\alpha|}\left(q_{\alpha}(x) \partial^{\alpha}\right)=0 \in g r^{F}\left(D_{n}(K)\right)
$$

portanto $q_{0}(x)=0$, além disso $q_{\alpha}(x) \partial^{\alpha} \in F_{|\alpha|-1}$ para cada $|\alpha| \geq 1$. Por tanto $q_{\alpha}(x)=0$ para $|\alpha| \geq 1$.

Consequentemente o polinômio $\sum_{\alpha} q_{\alpha}(y) z^{\alpha}$ é o polinômio zero e portanto a aplicação $\phi$ é injetiva.

Em resumo, $\phi$ define um isomorfismo de anéis. Isto prova nossa proposição.

Consideremos novamente a filtração ascendente $F_{i}=\left\{Q \in D_{n}(K): Q=\sum_{|\alpha| \leq i} q_{\alpha} \partial^{\alpha}\right\}$ do anel $D_{n}(K)$.

Definição 1.7. Seja $M$ um $D_{n}(K)$-módulo à esquerda. Uma família $\left\{\Gamma_{i}\right\}_{i \geq 0}$ de $K$-espaços vetoriais é uma filtração ascendente de $M$ se:

1. $\Gamma_{0} \subset \Gamma_{1} \subset \Gamma_{2} \subset \cdots \subset M$,

2. $M=\bigsqcup_{i \geq 0} \Gamma_{i}$,

3. $F_{i} \Gamma_{j} \subset \Gamma_{i+j}$, para todos os pares $i, j \geq 0$.

Suponhamos que $\Gamma=\left\{\Gamma_{i}\right\}_{i \geq 0}$ é uma filtração ascendente de um $D_{n}(K)$-módulo à esquerda $M$. Agora vamos construir um $g^{F}\left(D_{n}(K)\right)$ módulo graduado associado a esta filtração da seguinte maneira: Para cada $i \geq 0$, seja o $K$-espaço quociente $\Gamma(i):=\Gamma_{i} / \Gamma_{i-1}$, onde $\Gamma_{-1}:=0$. Consideremos a soma direta de eles:

$$
g r^{\Gamma}(M):=\Gamma(0) \oplus \Gamma(1) \oplus \Gamma(2) \oplus \cdots .
$$


Então $g r^{\Gamma}(M)$ é um $K$-espaço vetorial. Sobre $g r^{\Gamma}(M)$ definimos uma estrutura de $g^{F}\left(D_{n}(K)\right)$-módulo à esquerda como segue. Consideremos primeiro um elemento homogêneo $\sigma \in \Gamma(i)$ em $g r^{\Gamma}(M)$ e seja $f \in F(j)$ um elemento homogêneo em $g r^{F}\left(D_{n}(K)\right)$. Escolhemos $m \in \Gamma_{i}$ tal que sua imagem $\bar{m}$ no espaço quociente $\Gamma(i)$ é $\sigma$ e escolhemos $D \in F_{j}$ tal que $\bar{D}=f$. Agora $D m$ está em $\Gamma_{i+j}$. Definimos então $f \sigma$ como a imagem de $D m$ no espaço quociente $\Gamma(i+j)$.

De fato, temos que verificar $f \sigma$ está bem definido, ou seja que o elemento $f \sigma$ em $\Gamma(i+j)$ não depende das escolhas de $m$ e $D$. Para isto, notemos que se $m_{1} \in \Gamma_{i}$ é outro elemento tal que $\bar{m}_{1}=\sigma$ em $\Gamma(i)$, então a equação $\bar{m}=\bar{m}_{1}$ em $\Gamma(i)$ implica que $m-m_{1} \in \Gamma_{i-1}$ e por tanto $D m-D m_{1} \in \Gamma_{i+j-1}$, logo $\bar{D} \bar{m}=\bar{D} \bar{m}_{1}$ em $\Gamma(i+j)$. Analogamente podemos provar que $f \sigma$ não depende da escolha de $D$.

Finalmente, usando o fato que os elementos em $\operatorname{gr}^{F}\left(D_{n}(K)\right)$ e em $\operatorname{gr}^{\Gamma}(M)$ são somas finitas únicas de elementos homogêneos, podemos definir a $g r^{F}\left(D_{n}(K)\right)$-estrutura de módulo requerida.

Seja $M$ um $D_{n}(K)$-módulo à esquerda equipado com uma filtração ascendente $\Gamma$. Se $0 \neq m \in M$, então existe um único inteiro não negativo $k$ tal que $m \in \Gamma_{k} \backslash \Gamma_{k-1}$. Aqui lembremos que $\Gamma_{-1}:=0$. O inteiro não negativo $k$ é chamado de $\Gamma$-ordem de $m$ e a imagem $\bar{m}$ de $m$ em $\Gamma(k)$ é chamada de $\Gamma$-símbolo de $m$ e é denotada por $\gamma(m)$.

Proposição 1.8. Seja $\Gamma$ uma filtração ascendente sobre um $D_{n}(K)$-módulo à esquerda $M$. Suponhamos que $\left\{m_{\lambda}\right\}$ é um subconjunto de $M$ tal que $\left\{\gamma\left(m_{\lambda}\right)\right\}$ gera o gr ${ }^{F}\left(D_{n}(K)\right)$-módulo $g^{\Gamma}(M)$. Então $\left\{m_{\lambda}\right\}$ gera o $D_{n}(K)$-módulo $M$.

Prova: Desde que $\bigcup_{i \geq 0} \Gamma_{i}=M$ é suficiente provar que para cada $i \geq 0, \Gamma_{i}$ está contido no $D_{n}(K)$-submódulo gerado pelo conjunto $\left\{m_{\lambda}\right\}$. Usaremos indução sobre $i$ para provar isso. Suponhamos então que $\Gamma_{i-1} \subset \sum D_{n}(K) m_{\lambda}$. Agora seja $m \in \Gamma_{i} \backslash \Gamma_{i-1}$, então $\gamma(m) \in \Gamma(i)$. Pela hipótese podemos escrever $\gamma(m)=\sum f_{\lambda} \gamma\left(m_{\lambda}\right)$, onde a soma é finita e os $f_{\lambda} \in g r^{F}\left(D_{n}(K)\right)$. Se decompomos cada $f_{\lambda}$ em suas componentes homogêneas no anel graduado $g r^{F}\left(D_{n}(K)\right)$, obtemos uma soma finita da forma $f_{\lambda}=\sum f_{\lambda}(k)$ onde $f_{\lambda}(k) \in F(k)$.

Como $F(k) \Gamma(j) \subset \Gamma(k+j)$ para todo $j, k$, segue-se que $\gamma(m)=\sum f_{\lambda}\left(k_{\lambda}\right) \gamma\left(m_{\lambda}\right)$ onde $i=k_{\lambda}+\Gamma-$ ordem de $m_{\lambda}$.

Agora escolhemos $D_{\lambda} \in F_{k_{\lambda}}$ tal que $\bar{D}_{\lambda}=f_{\lambda}\left(k_{\lambda}\right)$. Então $m-\sum D_{\lambda} m_{\lambda}$ está em $\Gamma_{i-1}$, e como $\Gamma_{i-1} \subset \sum D_{n}(K) m_{\lambda}$ pela hipótese indutiva, concluimos que $m \in \sum D_{n}(K) m_{\lambda}$.

Definiçãa 1.9. Uma filtração ascendente $\Gamma$ sobre um $D_{n}(K)$-módulo à esquerda $M$ é uma boa filtração se $\mathrm{gr}^{\Gamma}(M)$ é finitamente gerado como um $\mathrm{gr}^{\mathrm{F}}\left(D_{n}(K)\right)$-módulo. 
Proposição 1.10. Se $M$ é um $D_{n}(K)$-módulo à esquerda que pode ser equipado com uma boa filtração, então $M$ é um $D_{n}(K)$-módulo finitamente gerado.

Prova: Seja $\Gamma$ uma boa filtração do módulo $M$ e seja $\sigma_{1}, \ldots, \sigma_{t}$ um conjunto finito de geradores de $g r^{\Gamma}(M)$ como $g r^{F}\left(D_{n}(K)\right)$-módulo. Decompomos cada $\sigma_{j}$ em seus componentes homogêneos, digamos $\sigma_{j}=\sum \sigma_{j}(k)$ onde $\sigma_{j}(k) \in \Gamma(k)$. Para cada par $j, k$ escolhemos $\gamma\left(m_{j k}\right)=\sigma_{j}(k)$. Vemos que $\left\{\gamma\left(m_{j k}\right)\right\}$ gera o $g r^{F}\left(D_{n}(K)\right)$-módulo $g r^{\Gamma}(M)$. A proposição 1.8 implica que o conjunto finito $\left\{m_{j k}\right\}$ gera $M$ como $D_{n}(K)$-módulo.

Proposição 1.11. $D_{n}(K)$ é um anel Noetheriano à esquerda, ou seja, todo ideal a esquerda é finitamente gerado.

Prova: Seja $I$ um ideal à esquerda em $D_{n}(K)$. Para cada $i \geq 0$ seja $\Gamma_{i}:=F_{i} \cap I$. Então é claro que $\Gamma_{i}$ define uma filtração ascendente sobre o $D_{n}(K)$-módulo $I$ e mais ainda $g r^{\Gamma}(I)$ é um ideal em $g r\left(D_{n}(K)\right)$. Mas $g r\left(D_{n}(K)\right)$ é um anel Noetheriano, pois pela proposição $\operatorname{gr}\left(D_{n}(K)\right)$ é isomorfo ao anel $K\left[\left[y_{1}, . ., y_{n}\right]\right]\left[z_{1}, \ldots, z_{n}\right]$. Portanto o ideal $g r^{\Gamma}(I)$ é finitamente gerado, em outras palavras, a filtração $\Gamma$ é uma boa filtração. A proposição 1.9 implica que $I$ é finitamente gerado.

Observação: Um resultado análogo é verdadeiro para ideais à direita. Ou seja, $D_{n}(K)$ é um anel Noetheriano à direita.

\subsection{Domínios de Ore}

Definição 1.12. Um domínio $R$ é um domínio de Ore à esquerda se $R x \cap R y \neq 0$ para todo par de elementos diferentes de zero $x, y \in R$. Analogamente, um domínio $R$ é um domínio de Ore à direita se $x R \cap y R \neq 0$ para todo par de elementos diferentes de zero $x, y \in R$. Um dominio R é um domínio de Ore se é um domínio de Ore à esquerda è direita.

Definição 1.13. Seja $R$ um dominio de integridade. Um anel de frações à esquerda para $R$ é um anel de divisão $D$ com as seguintes propriedades: $R$ é um subanel de $D$ e se $\delta \in D$, então existem $a \in R$ e $b \in R^{*}$ tais que $\delta=b^{-1} a$.

Proposição 1.14. Suponhamos que R é um domínio de Ore à esquerda, então existe um anel de frações à esquerda para $R$.

Prova: No conjunto $R \times R^{*}$ definimos uma relação $\sim$ como segue: $(a, b) \sim(c, d)$ se existem $r, s \in R^{*}$ tais que $r a=s c$ e $r b=s d \neq 0$. Então $\sim$ é uma relação de equivalência. De fato, a reflexividade e a simetria são claras. Por outro lado, se $(a, b) \sim(c, d)$ e $(c, d) \sim(e, f)$, então existem $r, s, t, u \in R^{*}$ tais que $r a=s c, r b=s d \neq 0, t c=u e$, 
$t d=u f \neq 0$; por outro lado, como $R$ é um domínio de Ore a esquerda temos que $x s=y t$ para alguns $x, y \in R^{*}, \operatorname{logo}(x r) a=(y u) e$ e $(x r) b=(y e) f \neq 0$ e portanto $(a, b) \sim(e, f)$, o que mostra que $\sim$ é transitiva.

Denotemos por $[a, b]$ a classe de equivalência do par $(a, b) \in R \times R^{*}$ e o conjunto quociente por $D:=R \times R^{*} / \sim$. Agora vamos a definir uma estrutura de anel sobre $D$ :

Dados $[a, b]$ e $[c, d]$ em $D$, escolhemos $r, s \in R^{*}$ tais que $r b=s d$ e colocamos $[a, b]+$ $[c, d]=[r a+s c, r b]$. Então + é uma operação bem definida.

Por outro lado, dados $[a, b]$ e $[c, d]$ em $D$, escolhemos $r \in R^{*}$ e $s \in R$ tais que $r a=s d$, e colocamos $[a, b] \cdot[c, d]=[s c, r b]$. Então $\cdot$ é uma operação bem definida.

O sistema $(D,+, \cdot)$ é um anel, onde $[0,1]$ é o elemento zero e $[1,1]$ é o elemento unitário de $D$. Mais ainda a aplicação $r \mapsto[r, 1]$ é um homomorfismo injetivo de anéis. Com efeito, o fato de ser homomorfismo segue-se das definições e por outro lado, se $[r, 1]=$ $[0,1]$ para algum $r \in R$ temos que $(r, 1) \sim(0,1)$ e por tanto existem $t, u \in R^{*}$ tais que $t r=s \cdot 0=0$ e como $R$ é um domínio, concluímos que $r=0$. O homomorfismo injetivo anterior permite identificar $R$ com um subanel de $D$ e quando fazemos esta identificação, $R$ torna-se um subanel de $D$.

Além disso, o anel $D$ é um anel de divisão. De fato, se $[a, b]$ é um elemento diferente de zero em $D$, então $a, b \in R^{*}, \operatorname{logo}[b, a] \in D$, mais ainda $[a, b] \cdot[b, a]=[1,1]$ e $[b, a] \cdot[a, b]=[1,1]$. Notemos que se $r \in R^{*}$, então $[r, 1]^{-1}=[1, r]$. Usaremos a notação $r^{-1}$ para o inverso de $r \in R^{*}$.

Finalmente, se $\delta \in D$, então existem $a \in R$ e $b \in R^{*}$ tais que $\delta=[a, b]$, que pode ser escrito como $[a, b]=[b, 1] \cdot[a, 1]=[b, 1]^{-1} \cdot[a, 1]$. Logo, se usamos a identificação de $R$ como um subanel de $D$ e a notação acima, temos que $\delta=b^{-1} a$.

Proposição 1.15. Seja $R$ um domínio de Ore à esquerda e $D$ um anel de frações à esquerda para $R$. Suponhamos que $\phi: R \rightarrow S$ é um homomorfismo de anéis tal que $\phi(x)$ é uma unidade de $S$ para cada $x \in R^{*}$. Então $\phi$ estende-se de maneira única a um homomorfismo de anéis $\psi: D \rightarrow S$.

Prova: Seja $\delta \in D$, então existem $a \in R$ e $b \in R^{*}$ tais que $\delta=b^{-1} a$. Colocamos $\psi(\delta)=$ $\phi(b)^{-1} \phi(a)$; em primeiro lugar temos que ver que $\psi$ é uma aplicação bem definida. Com efeito, suponhamos que $\delta$ tem outra representação como $\delta=d^{-1} c$ para alguns $c \in R$ e $d \in R^{*}$. Então $b^{-1} a=d^{-1} c$ e como $R$ é um domínio de Ore a esquerda, existem $r, s \in R^{*}$ tais que $r a=s c$ e $r b=s d \neq 0$. Mas $\phi(r b), \phi(r), \phi(s d), \phi(s)$ são unidades em $S$. Logo $\phi(b)^{-1} \phi(a)=\phi(r b)^{-1} \phi(r a)=\phi(s d)^{-1} \phi(s c)=\phi(d)^{-1} \phi(c)$. Por tanto a regra $\psi\left(b^{-1} a\right)=\phi(b)^{-1} \phi(a)$ define uma aplicação $\psi: D \rightarrow S$. Notemos que $\phi(r)=\psi\left(1^{-1} r\right)=\phi(1)^{-1} \phi(r)=\phi(r)$ para todo $r \in R$, por tanto $\psi$ estende $\phi$ a $D$. Em particular $\psi(1)=1$. 
Agora mostremos que de fato $\phi$ é um homomorfismo de anéis. Com efeito, sejam $a, c \in R$ e $b, d \in R^{*}$, então existem $r, s \in R^{*}$ tais que $r b=s d$. Logo

$\psi\left(b^{-1} a+d^{-1} c\right)=\psi\left((r b)^{-1}(r a+s c)\right)=\phi(r b)^{-1} \phi(r a+s c)=\phi(r b)^{-1} \phi(r a)+\phi(r b)^{-1} \phi(s c)=$ $\phi(b)^{-1} \phi(a)+\phi(d)^{-1} \phi(c)=\psi\left(b^{-1} a\right)+\psi\left(d^{-1} c\right)$.

Por outro lado, existem $t \in R$ e $u \in R^{*}$ tais que $u a=t d$. Logo

$\psi\left(b^{-1} a d^{-1} c\right)=\psi\left((u b)^{-1} t c\right)=\phi(u b)^{-1} \phi(t c)=\phi(b)^{-1} \phi\left(u^{-1} t\right) \phi(c)=\phi(b)^{-1} \phi\left(a d^{-1}\right) \phi(c)=$ $\left(\phi(b)^{-1} \phi(a)\right)\left(\phi(d)^{-1} \phi(c)\right)=\psi\left(b^{-1} a\right) \psi\left(d^{-1} c\right)$.

Por tanto $\psi$ é um homomorfismo de anéis.

Finalmente, si $\varphi: D \rightarrow S$ é outro homomorfismo de anéis que estende $\phi$, temos que para $a \in R$ e $b \in R^{*}, \varphi\left(b^{-1} a\right)=\varphi(b)^{-1} \varphi(a)=\phi(b)^{-1} \phi(a)=\psi\left(b^{-1} a\right)$. Por tanto $\varphi=\psi, \mathrm{o}$ que mostra a unicidade da extensão.

Corolário 1.16. Seja $R$ um domínio de Ore à esquerda e sejam $D, D^{\prime}$ anéis de frações à esquerda para $R$. Então a aplicação identidade de R estende-se a um único isomorfismo de D sobre $D^{\prime}$.

No sentido do corolário anterior, um anel de frações à esquerda para um domínio de Ore à esquerda é único.

Definição 1.17. Seja $R$ um domínio de integridade. Um anel de frações à direita para $R e ́$ um anel de divisão $E$ com as seguintes propriedades: $R$ é um subanel de $E$ e se $\xi \in E$, então existem $a \in R$ e $b \in R^{*}$ tais que $\xi=a b^{-1}$.

É claro que proposições semelhantes são verdadeiras para domínios de Ore à direita.

Proposição 1.18. Suponhamos que $R$ é um domínio de Ore à direita, então existe um anel de frações à direita para $R$.

Proposição 1.19. Seja $R$ um domínio de Ore à direita e $E$ um anel de frações à direita para $R$. Suponhamos que $\phi: R \rightarrow S$ é um homomorfismo de anéis tal que $\phi(x)$ é uma unidade de $S$ para cada $x \in R^{*}$. Então $\phi$ estende-se de maneira única a um homomorfismo de anéis $\psi: E \rightarrow S$.

Corolário 1.20. Seja $R$ um dominio de Ore à direita e sejam $E, E^{\prime}$ anéis de frações à direita para $R$. Então a aplicação identidade de R estende-se a um único isomorfismo de E sobre $E^{\prime}$.

No sentido do corolário anterior, um anel de frações à direita para um domínio de Ore à direita é único.

Proposição 1.21. Suponhamos que $R$ é um dominio de Ore. Então os anéis de frações à esquerda e à direita são iguais. 
Prova: Seja $D$ o anel de frações à esquerda de $R$ e $E$ o anel de frações à direita de $R$. Então se $\delta \in D$, existem elementos $a \in R$ e $b \in R^{*}$ tais que $\delta=b^{-1} a$. Desde que $R$ é um domínio de Ore à direita, existem $r \in R$ e $s \in R^{*}$ tais que $a s=b r, \operatorname{logo} \delta=r s^{-1} \mathrm{e}$ por tanto $\delta \in E$, o que mostra que $D \subset E$. Analogamente pode-se provar que $E \subset D$ e por tanto $D=S$.

Proposição 1.22. Seja $R$ um domínio. Então ou $R$ é um domínio de Ore à esquerda ou $R$ contém um ideal à esquerda que é livre e de posto infinito como $R$-módulo. Em particular, todo dominio Noetheriano à esquerda é um dominio de Ore à esquerda.

Prova: Suponhamos que $R$ não é um domínio de Ore à esquerda. Então existem $a, b \in R^{*}$ tais que $R a \cap R b=0$.

Afirmação: Os elementos $a, a b, a b^{2}, a b^{3}, \ldots$, são linearmente independentes à esquerda sobre $R$, logo o ideal à esquerda gerado por eles é livre de posto infinito. De fato, caso contrário, haveria uma relação $\sum c_{i} a b^{i}$, onde os $c_{i}$ não são todos iguais a zero. Seja $c_{r} \mathrm{o}$ primeiro coeficiente diferente de zero, então podemos cancelar $b^{r}$ e obter uma relação

$$
c_{r} a+c_{r+1} a b+\cdots+c_{n} a b^{n-r}=0 .
$$

Donde

$$
\left(c_{r+1} a+\cdots+c_{n} a b^{n-r-1}\right) b=-c_{r} a .
$$

O que contradiz à hipótese inicial sobre $a$ e $b$.

Um resultado análogo é válido para "direita"no lugar de "esquerda".

Proposição 1.23. Seja $R$ um domínio. Então ou $R$ é um dominio de Ore à direita ou $R$ contém um ideal à direita que é livre e de posto infinito como R-módulo. Em particular, todo domínio Noetheriano à direita é um domínio de Ore à direita.

Corolário 1.24. Todo dominio Noetheriano é um domínio de Ore. Em particular $D_{n}(K)$ é um dominio de Ore. 


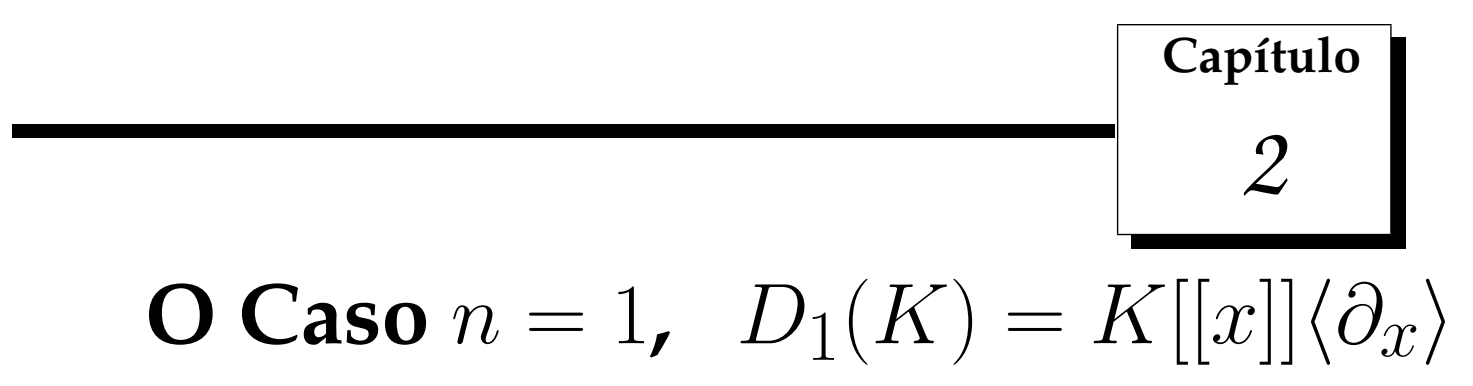

Neste capítulo estudaremos as bases de divisão do anel $D_{1}(K)$ e provaremos, usando este conceito, que todo ideal à esquerda de $D_{1}(K)$ é dois gerado. A principal referência é [6].

\subsection{Bases de Divisão}

Seja $K$ um corpo de característica zero e seja $P \in D_{1}(K)=K[[x]]\left\langle\partial_{x}\right\rangle, P \neq 0$. Então existem $a_{0}(x), a_{1}(x), \ldots, a_{d}(x) \in K[[x]] \operatorname{com} a_{d}(x) \neq 0$ tais que

$$
P=a_{d}(x) \partial_{x}^{d}+\cdots+a_{1}(x) \partial_{x}+a_{0}(x)
$$

O inteiro não negativo $d$ é chamado de ordem do operador $P$ e é denotado por $\operatorname{ord}(P)$.

Por definição o expoente de $P$ é o par de inteiros

$$
\exp (P)=\left(v\left(a_{d}\right), d\right) \in \mathbb{N}^{2}
$$

onde $d=\operatorname{ord}(P)$ é a ordem do operador $P$ e $v\left(a_{d}\right)$ é a valuação da série $a_{d}(x)$. Tal expoente é aditivo com respeito ao produto, ou seja, se $Q \in D_{1}(K)$ é outro operador diferente de zero, então

$$
\exp (P Q)=\exp (P) \exp (Q) \in \mathbb{N}^{2}
$$

De fato, se $Q=b_{e}(x) \partial_{x}^{e}+\cdots+b_{0}(x) \operatorname{com} b_{e}(x) \neq 0$, então podemos escrever

$$
P Q=a_{d} b_{e} \partial_{x}^{d+e}+R \operatorname{com} \operatorname{ord}(R)<d+e
$$


Proposição 2.1. Sejam A e P elementos diferentes de zero de $D_{1}(K) \operatorname{com} \exp (P)=(v, d)$. Então existe um unico par $(Q, R)$ de elementos de $D_{1}(K)$ tais que

1. $A=P Q+R$

2. $R=\sum \sum u_{k, l}(x) x^{k} \partial_{x}^{l}+S$ onde $u_{k, l}$ são unidades de $K[[x]]$ e ord $(S)<d$.

Prova: A prova da proposição é feita por indução sobre a ordem do operador $A$.

Definição 2.2. Seja I um ideal à esquerda de $D_{1}(K)$. O conjunto Exp $(I)$ é definido como:

$$
\operatorname{Exp}:=\left\{\exp (P): P \in D_{1}(K), P \neq 0\right\}
$$

Desde que $I$ é um ideal à esquerda de $D_{1}(K)$ temos que

$$
\operatorname{Exp}(I)=\operatorname{Exp}(I)+\mathbb{N}^{2} .
$$

Seja $r$ a ordem mínima dos elementos diferentes de zero do ideal a esquerda $I$ e seja $A_{r}$ o conjunto dos operadores de $D_{1}(K)$ com ordem igual a $r$. Cada $P_{r} \in A_{r}$ pode ser escrita de maneira única como $P_{r}=a_{r, P_{r}}(x) \partial_{x}^{r}+$ (termos de menor ordem). Escolhemos

$\alpha_{r}:=\min \left\{v\left(a_{r, P_{r}}(x)\right): P_{r} \in A_{r}\right\}$. Então o par $\left(\alpha_{r}, r\right) \in \operatorname{Exp}(I)$. Seja agora $A_{r+1} \mathrm{o}$ conjunto dos operadores de $D_{1}(K)$ com ordem igual a $r+1$. De maneira semelhante, escolhemos $\alpha_{r+1}:=\min \left\{v\left(a_{r+1, P_{r+1}}(x)\right): P_{r+1} \in A_{r+1}\right\}$. Notemos que $\alpha_{r+1} \leq \alpha_{r}$, pois se $P_{r}$ é tal que $\exp \left(P_{r}\right)=\left(\alpha_{r}, r\right)$, então $P_{r}=u x^{\alpha_{r}} \partial_{x}^{r}+\cdots \in I$, onde $u$ é uma unidade de $K[[x]]$. Usando o fato que $I$ é um ideal a esquerda temos que $\partial_{x} u^{-1} P_{r} \in I$, mas $\partial_{x} u^{-1} P_{r}=x^{\alpha_{r}} \partial_{x}^{r+1}+\left(\right.$ termos de menor ordem), logo pela definição de $\alpha_{r+1}$, concluimos que $\alpha_{r+1} \leq \alpha_{r}$. O par $\left(\alpha_{r+1}, r+1\right) \in \operatorname{Exp}(I)$. Continuando com este processo obtemos um conjunto $\left\{\left(\alpha_{r}, r\right),\left(\alpha_{r+1}, r+1\right), \ldots,\right\}$ tais que em geral $\alpha_{j}$ é a valuação minimal dos elementos de $I$ que tem ordem $j$. Como $\alpha_{r} \geq \alpha_{r+1} \geq \cdots$ em $\mathbb{N}$, é claro que este processo acaba digamos em $\left(\alpha_{s}, s\right)$.

Definição 2.3. Os pontos $\left(\alpha_{r}, r\right),\left(\alpha_{r+1}, r+1\right), \ldots,\left(\alpha_{s}, s\right)$ são chamados pontos escada e $o$ conjunto formado por eles será denotado por Es(I). Ou seja,

$$
E s(I)=\left\{\left(\alpha_{r}, r\right),\left(\alpha_{r+1}, r+1\right), \ldots,\left(\alpha_{s}, s\right)\right\}
$$

Usando a divisão acima podemos ver que o conjunto $E s(I)$ é o conjunto de pontos fronteira de $\operatorname{Exp}(I)$. 
Das definições anteriores temos a igualdade:

$$
\operatorname{Exp}(I)=\bigcup_{p \leq j \leq q}\left\{\left(\alpha_{j}, j\right)+\mathbb{N} \times\{0\}\right\} \cup\left\{\left(\alpha_{q}, q\right)+\mathbb{N}^{2}\right\}
$$

Definição 2.4. Seja I um ideal a esquerda de $D_{1}(K)$. Uma base de divisão de I consiste de todos os elementos $P_{j} \in I$ tais que exp $\left(P_{j}\right)=\left(\alpha_{j}, j\right)$ para algum $\left(\alpha_{j}, j\right) \in \operatorname{Es}(I)$.

A terminologia anterior provem da seguinte :

Proposição 2.5. Seja I um ideal a esquerda próprio de $D_{1}(K)$ e sejam $P_{r}, \ldots, P_{s}$ uma base de divisão para $I$

1. Para todo $A \in D_{1}(K)$ existem únicos elementos $Q_{r}, Q_{r+1}, \ldots, Q_{s-1} \in K[[x]]$, $Q_{s} \in D_{1}(K)$ e $R \in D_{1}(K)$ tais que

$$
A=Q_{r} P_{r}+\cdots+Q_{s} P_{s}+R
$$

com

$$
R=\sum_{l=r}^{\operatorname{ord}(A)} \sum_{k=0}^{\alpha_{l}-1} u_{k, l} x^{k} \partial_{x}^{l}+S
$$

$e \operatorname{ord}(S)<r$.

2. Com essas notações temos que $A \in I$ se e somente se $R=0$

Prova: A existência de tal divisão provém da divisão estabelecida na proposição 2.1. Primeiro dividimos $A$ por $P_{s}$, em seguida dividimos o resíduo que é de ordem menor que $s$ por $P_{s-1}$, e assim por diante.

Mais ainda tem-se que $A \in I$ se e somente se $R \in I$. Mas se $R \neq 0$ temos que $\exp (R) \notin \operatorname{Exp}(I), \log \mathrm{E} \in I$ se e somente se $R=0$. Isto dá a segunda parte da proposição e também a unicidade de $R$.

A fim de provar a unicidade dos $Q_{r}, \ldots, Q_{s}$ é suficiente provar que se

$$
Q_{r} P_{r}+\cdots+Q_{s} P_{s}=0
$$

com $Q_{r}, \ldots, Q_{s-1} \in K[[x]]$ e $Q_{s} \in D_{1}(K)$, então todos esses operadores são zero. Isto pode ser mostrado considerando o termo de maior ordem de dita soma.

Corolário 2.6. Sejam $I$ e $I^{\prime}$ ideais de $D_{1}(K)$ tais que $I^{\prime} \subset I \operatorname{com} E s\left(I^{\prime}\right)=E s(I)$. Então $I^{\prime}=I$. 
Prova: Neste caso uma base de divisão $P_{r}^{\prime}, \ldots, P_{s}^{\prime}$ para $I^{\prime}$ é também uma base de divisão para . O critério para que um operador pertencer a $I^{\prime}$ é o mesmo que para $I$, em virtude da proposição anterior.

\subsection{Os ideais á esquerda de $D_{1}(K)$ são dois gerados.}

A continuação mostraremos que todo ideal à esquerda $I$ é dois gerado. Antes precisaremos de dois lemas

Lema 2.7. Seja I um ideal à esquerda de $D_{1}(K)$ e seja $P_{r}, \ldots, P_{s}$ uma base de divisão para $I$. Então para todo $A \in I$ existe $k$ tal que $x^{k} A \in D_{1}(K)$. Ou seja o $D_{1}(K)$-módulo à esquerda $I / D_{1}(K) P_{r}$ é um módulo de torção.

Prova: A fim de provar o lema, observemos que na divisão elementar de $A$ por $P_{r}$, se temos que $\exp (A) \in \exp (P)+\mathbb{N}^{2}$, então o resíduo $R$ tem ordem menor que a $\operatorname{ord}(A)$. Escolhemos $n_{1}$ tal que $\exp \left(x^{n_{1}} A\right) \in \exp \left(P_{r}\right)+\mathbb{N}^{2}$. Então temos que

$$
x^{n_{1}} A=B_{1} P_{r}+R_{1}, \quad \operatorname{ord}\left(R_{1}\right)<\operatorname{ord}(A)
$$

e por indução achamos $n$ tal que

$$
x^{n} A=B P_{r}+R
$$

$\operatorname{com} \operatorname{ord}(R)<\operatorname{ord}\left(P_{r}\right)=r$. Mas $R \in I$ e $\operatorname{assim} R=0$.

Lema 2.8. Seja $M$ um $D_{1}(K)$-módulo à esquerda finitamente gerado que é também finitamente gerado como $K[[x]]$-módulo. Então $M$ é um $K[[x]]$-módulo livre.

Prova: Como $M$ é finitamente gerado como $K[[x]]$-módulo, então o $K$-espaço vetorial $M / x M$ tem dimensão finita. Seja $m_{1}+x M, \ldots, m_{n}+x M$ uma base deste espaço vetorial. Então se $N$ é o $K[[x]]$-submódulo de $M$ gerado pelos elementos $m_{1}, . ., m_{n} \in M$, temos a igualdade $M=N+x M$. Usando o lema de Nakayama temos que $N=M$, ou seja $m_{1}, \ldots, m_{n}$ é um conjunto de geradores de $M$ sobre $K[[x]]$. Portanto obtém-se uma aplicação sobrejetiva $K[[x]]$-linear $\phi: K[[x]]^{n} \rightarrow M$. Seja $L$ o núcleo de $\phi$. Mostraremos que $L=0$ e por tanto $\phi$ será um isomorfismo, o que provará nosso lema. Com efeito, suponhamos que $L \neq 0$. Seja $v \in L, v \neq 0$, então existem séries $p_{1}(x), \ldots, p_{n}(x) \in K[[x]]$ não todas iguais a zero tais que $v=\sum_{i=1}^{n} p_{i}(x) \varepsilon_{i}$, onde $\varepsilon_{1}, \ldots, \varepsilon_{n}$ é a base canônica de $K[[x]]^{n}$, mais ainda $\phi(v)=\sum_{i=1}^{n} p_{i}(x) m_{i}=0$ em $M$. 
Como $M$ é também um $D_{1}(K)$-módulo, temos que $\partial_{x}\left(\sum_{i=1}^{n} p_{i}(x) m_{i}\right)=0$ em $M$. Então $\left.\sum_{i=1}^{n}\left(\partial_{i} p_{i}(x)\right) m_{i}=\sum_{i=1}^{n}\left(\partial p_{i}(x) / \partial x+p_{i} \partial_{i}\right) m_{i}=\sum_{i=1}^{n}\left(\partial p_{i}(x) / \partial x\right) m_{i}+\sum_{j=1}^{n} p_{j}(x)\left(\partial_{x} m_{j}\right)\right)=0$

por outro lado $\partial_{x} m_{j}=\sum_{i=1}^{n} b_{j i}(x) m_{i}$ para algumas $b_{j i}(x) \in K[[x]]$. Então temos $\left.\sum_{i} \partial p_{i}(x) / \partial_{x} m_{i}+\sum_{j=1}^{n} p_{j}(x)\left(\sum_{i=1}^{n} b j i\right) m_{i}\right)=\sum_{i} \partial p_{i}(x) / \partial_{x} m_{i}+\sum_{i=1}^{n}\left(\sum_{j=1}^{n} p_{j}(x) b_{i j}(x)\right) m_{i}$ $=\sum_{i=1}^{n}\left(\partial p_{i}(x) / \partial_{x}+\sum p_{j}(x) b_{j i}(x)\right) m_{i}=0$ e por tanto temos que $\sum_{i}^{n}\left(\partial p_{i}(x) / \partial_{x}+\sum_{j} p_{j}(x) b j i(x)\right) \varepsilon_{i} \in L$. Sem perda de generalidade suponhamos que $p_{1}(x) \neq 0$ é que $r=\operatorname{val}\left(p_{1}(x)\right)$ é a menor valuação. Então continuando com o processo anterior e depois de $r$ etapas obtemos uma igualdade da forma $\left(\partial^{r} p_{1}(x) / \partial x^{r}\right) \varepsilon_{1}+$ $\sum_{j=2}^{n} q_{j}(x) \varepsilon_{j} \in L$. Logo se trabalhamos no $K$-espaço quociente $M / x M$, obtemos uma igualdade do tipo

$$
\left(\partial^{r} p_{1}(x) / \partial x^{r}\right)+\sum_{j=2}^{n} p_{j}(x) q_{j}(x)=0 .
$$

Mas $\operatorname{val}\left(\partial^{r} p_{1}(x) / \partial x^{r}\right)=0, \operatorname{logo} 0=\operatorname{val}\left(\partial^{r} p_{1}(x) / \partial x^{r}\right)=\operatorname{val}\left(\sum_{j=2}^{n} p_{j}(x) q_{j}(x)\right) \geq \min \left\{\operatorname{val}\left(p_{j}(x) q_{j}(x)\right)\right\}$, o que é uma contradição, pois por nossa escolha $\operatorname{val}\left(p_{1}(x)\right) \geq \operatorname{val}\left(p_{j}(x)\right)$ para todo $j \neq 1$. Concluímos então que $L=\operatorname{Ker}(\phi)=0$.

Agora podemos provar que todo ideal à esquerda de $D_{1}(K)$ é 2-gerado.

Teorema 2.9. Seja I um ideal a esquerda de $D_{1}(K)$ e seja $P_{r}, \ldots, P_{s}$ uma base de divisão para I. Então I é gerado por apenas $P_{r}$ e $P_{s}$.

Prova: Seja $J:=D_{1}(K) P_{r}+D_{1}(K) P_{s} \subset I$, o ideal à esquerda gerado por $P_{r}$ e $P_{s}$. Consideremos o $D_{1}(K)$-módulo à esquerda $I / J$. Como o $D_{1}$-módulo $I / D_{1}(K) P_{r}$ é de torção segundo o lema 2.7 , então $I / J$ é também de torção. Por outro lado $I / J$ é um módulo finitamente gerado sobre $D_{1}(K)$, mais ainda é finitamente gerado como $K[[x]]$-módulo, na verdade é gerado pelas classes de $P_{r+1}, \ldots, P_{s-1}$ devido à proposição 2.5. Então pelo lema $2.8, I / J$ é um $K[[x]]$-módulo livre. Assim, $I / J$ é de torção e livre como $K[[x]]$-módulo. Então $I / J=0, \operatorname{logo} I=J$. 


\section{Capítulo}

\section{Módulos Livres sobre $S=T((x))\left\langle\partial_{x}\right\rangle \mathbf{e}$ sobre $D=F[[x]]\left\langle\partial_{x}\right\rangle$}

Neste capítulo estudamos algumas proposições referentes a módulos livres sobre os anéis $S=T((x))\left\langle\partial_{x}\right\rangle$ e $D=F[[x]]\left\langle\partial_{x}\right\rangle$. Na primeira seção definimos e exploramos algumas propriedades desses anéis. As proposições sobre módulos livres que precisaremos no capítulo seguinte são estudadas na segunda seção. A proposição 3.10 é a que será realmente utilizada adiante, mas o resultado técnico mais importante é a proposição 3.6.

\subsection{Propriedades dos anéis $S$ e $D$}

\subsubsection{O anel $S=T((x))\left\langle\partial_{x}\right\rangle$}

Seja $T$ um anel de divisão de característica zero. Por $T[[x]]$ denotamos o anel de séries de potências em uma indeterminada com coeficientes em $T$, onde $x t=t x$ para todo $t \in T$. Como $T$ é um domínio Noetheriano à esquerda e à direita, então $T[[x]]$ é também um domínio Noetheriano à esquerda e à direita (veja por exemplo [11], theorem 4.5, chapter 1 , página 22). Logo, pelo corolário 1. 23, $T[[x]]$ é um domínio de Ore à esquerda e à direita. Por tanto, $T[[x]]$ possui um anel de frações que denotamos por $T((x))$.

Sobre $T[[x]]$ temos a derivação $T$-linear usual $\partial_{x}: T[[x]] \rightarrow T[[x]]$. Vamos estender esta derivação a uma derivação $T$-linear sobre o anel de frações $T((x))$ da seguinte maneira: Seja $\delta \in T((x))$, então existem $a, b \in T[[x]] \operatorname{com} b \neq 0$ tais que $\delta=b^{-1} a$. Consideremos 
a aplicação:

$$
D_{x}: T((x)) \rightarrow T((x)), \text { onde } D_{x}(\delta)=b^{-1} \partial_{x}(a)-b^{-1} \partial_{x}(b) \delta
$$

Não é difícil ver que tal aplicação está bem definida, ou seja, não depende dos representantes $a, b$ da fração $\delta$. Mais ainda, $D$ é uma derivação $T$-linear que estende $\partial_{x}$; é por isso que usaremos a notação $\partial_{x}$ em lugar de $D_{x}$.

Por outro lado, se $\delta \in T((x))$, então $\delta$ define um operador $T$-linear sobre $T((x))$ que leva um elemento $\gamma \in T((x))$ no produto $\delta \gamma$.

Definição 3.1. O subanel dos T-operadores lineares de $T((x))$ que é gerado pelos elementos de $T((x))$ e a derivação $\partial_{x}$ é chamado anel de operadores diferenciais T-lineares de $T((x))$, ou simplesmente o anel de operadores diferenciais de $T((x))$. Denotamos este anel por $S=T((x))\left\langle\partial_{x}\right\rangle$.

Seja $\delta$ um elemento de $T((x))$, então em $S$ temos a seguinte regra de comutação entre $\partial_{x}$ e operador produto definido por $\delta$ :

$$
\left[\partial_{x}, \delta\right]=\partial_{x}(\delta)
$$

Em virtude desta última relação podemos mostrar, como no caso de $D_{1}(K)$, que todo elemento diferente de zero $\alpha \in S$ pode ser escrita de maneira única como:

$$
\alpha=p_{0}+p_{1} \partial_{x}+\cdots+p_{k} \partial^{k}
$$

onde $k$ é um inteiro não negativo, $p_{0}, p_{1}, \ldots, p_{k} \in T((x))$ e $p_{k} \neq 0$. O inteiro $k$ é chamado de ordem de $\alpha$ de $\alpha$ e é denotado por $\operatorname{ord}(\alpha)$.

Notemos que se $\beta$ é outro elemento de $S$ diferente de zero de ordem $l$, então $\operatorname{ord}(\alpha \beta)=$ $k+l$. Portanto $S$ é um domínio de integridade. Do mesmo modo que $D_{1}(K), S$ é um anel simples.

Proposição 3.2. (Algoritmo da Divisão em $S$ ). Sejam $\alpha, \beta \in S \operatorname{com} \beta \neq 0$ então existem $\gamma, \theta \in S$ tais que

$$
\alpha=\gamma \beta+\theta, \operatorname{com} \theta=0 \text { ou } \operatorname{ord}(\theta)<\operatorname{ord}(\beta) .
$$

Prova: Se $\alpha=0$, basta tomar $\gamma=\theta=0$. Suponhamos então que $\alpha \neq 0$ e que

$$
\alpha=p_{0}+p_{1} \partial_{x}+\cdots+p_{k} \partial^{k}, \beta=q_{0}+q_{1} \partial_{x}+\cdots+q_{n} \partial^{n}, \text { com } p_{k} \neq 0 \text { e } q_{n} \neq 0
$$

Fazemos indução sobre $k=\operatorname{ord}(\alpha)$. Se $k=0$ e $n>0$, escolhemos $\gamma=0$ e $\theta=\alpha$. Se $k=n=0$, escolhemos $\gamma=p_{0} q_{0}^{-1}$ e $\theta=0$. 
Suponhamos que a proposição é verdadeira para operadores de ordem menor que $k$. Sem perda de generalidade podemos supor que $n \leq k$, pois caso contrário basta tomar $\gamma=0$ e $\theta=\alpha$. Consideremos o operador $\alpha_{1}=\alpha-p_{k} q_{n}^{-1} \partial_{x}^{k-n}$, então $\operatorname{ord}\left(\alpha_{1}\right)<k$. Logo pela hipótese indutiva, existem $\gamma_{1}$ e $\theta$ em $S$ tais que

$$
\alpha_{1}=\gamma_{1} \beta+\theta, \text { onde } \theta=0 \text { ou } \operatorname{ord}(\theta)<\operatorname{ord}(\beta) .
$$

$\operatorname{Logo} \alpha=\gamma \beta+\theta$, onde $\gamma=\gamma_{1}+p_{k} q_{n}^{-1} \partial_{x}^{k-n}$ e $\theta=0 \quad$ ou $\operatorname{ord}(\theta)<\operatorname{ord}(\beta)$.

Corolário 3.3. Todo ideal à esquerda de S é principal.

Prova: Seja $I$ um ideal a esquerda diferente de zero de $S$. Escolhemos em $I$ um operador $\beta \neq 0$ com a menor ordem. Afirmamos que o ideal $I$ é gerado por $\beta$. Com efeito, seja $\alpha$ um elemento qualquer de $I$. Então pela proposição anterior, existem $\gamma, \theta \in S$ tais que $\alpha=\gamma \beta+\theta$ onde $\theta=0$ ou $\operatorname{ord}(\theta)<\operatorname{ord}(\beta)$. Como $I$ é um ideal a esquerda e $\alpha, \beta \in I$ temos que $\theta$. Então pela escolha de $\beta$ concluímos que $\theta=0$ e por tanto $\alpha=\gamma \beta$.

Proposição 3.4. Seja $0 \neq \alpha \in S$. Então o $S$-módulo $S / S \alpha$ tem comprimento finito.

Prova: Seja $k$ a ordem de $\alpha$. Então podemos escrever $\alpha=p_{0}+\cdots+p_{k} \partial^{k}, p_{i} \in T((x))$ e $p_{k} \neq 0$. Como $T((x))$ é um anel de divisão segue-se que $S \alpha$ contém $p_{k}^{-1} \alpha=q_{0}+\cdots+$ $q_{k-1} \partial^{k-1}+\partial^{k}$. Pelo algoritmo da divisão em $S$, dado $\beta \in S$, existem $\gamma, \theta \in S$ tais que $\beta=\gamma \alpha+\theta$ onde $\theta=0$ ou $\operatorname{ord}(\theta)<k$, ou seja, $\theta=r_{0}+\cdots+r_{k-1} \partial^{k-1}$, para alguns $r_{i} \in T((x))$. Então $S / S \alpha$ é um espaço vetorial sobre $T((x))$ de dimensão $k$. Por outro lado cada $S$-submódulo de $S / S \alpha$ induz um $T((x))$-subespaço de $S / S \alpha$. Por tanto o $S$-módulo $S / S \alpha$ tem comprimento no máximo $k$.

\subsubsection{O anel $D=F[[x]]\left\langle\partial_{x}\right\rangle$}

Seja $F$ um corpo de característica zero e $D=D_{1}(F)$.

Proposição 3.5. Seja a um elemento diferente de zero de D. Então o D-módulo quociente $D / D \alpha$ tem comprimento finito.

Prova: Como $\alpha$ é um elemento diferente de zero de $D$, o $D$-módulo quociente $D / D \alpha$ é um $D$-módulo holonômico. Portanto $D / D \alpha$ tem comprimento finito. (Veja [6], página 14.) 


\subsection{Módulos livres}

Suponhamos que $F$ é um corpo de característica zero contido em $T$.

Proposição 3.6. (i) Sejam $\delta_{1}, \ldots, \delta_{m}$ elementos diferentes de zero de $D=F[[x]]\left\langle\partial_{x}\right\rangle \subset S$. Seja $\alpha \in S, \alpha \neq 0$ um elemento de $S$ e $S^{m}=S \varepsilon_{1}+\cdots+S \varepsilon_{m}$ um $S$-módulo livre de posto $m$ com base $\varepsilon_{1}, \ldots, \varepsilon_{m}$. Seja $M$ o $S$-submódulo de $S^{m}$ gerado pelo conjunto $\left\{\alpha \delta_{1} f \varepsilon_{1}+\cdots+\alpha \delta_{m} f \varepsilon_{m} / f \in \mathbb{Z}[[x]]\left\langle\partial_{x}\right\rangle\right\}$. Então $M=S^{m}$.

(ii) Sejam $\delta_{1}, \ldots, \delta_{m}$ elementos diferentes de zero de $D=F[[x]]\left\langle\partial_{x}\right\rangle$. Seja $0 \neq \alpha$ um elemento de $D$ e $D^{m}=D \varepsilon_{1}+\cdots+D \varepsilon_{m}$ um D-módulo livre de posto $m$ com base $\varepsilon_{1}, \ldots, \varepsilon_{m}$. Seja $M$ o D-submódulo de $D^{m}$ gerado pelo conjunto $\left\{\alpha \delta_{1} f \varepsilon_{1}+\cdots+\alpha \delta_{m} f \varepsilon_{m} / f \in \mathbb{Z}[[x]]\left\langle\partial_{x}\right\rangle\right\}$. Então $M=D^{m}$.

Prova: Vamos mostrar $(i)$. A prova de $(i i)$ é análoga.

Para simplificar a notação colocamos $\partial:=\partial_{x}$.

Primeiro observemos que a hipótese e a tese não mudam se a $m$-upla $\delta_{1}, \ldots, \delta_{m}$ for substituída por uma $m$-upla $\beta_{1}, \ldots, \beta_{m}$, onde $\beta_{i}=\Sigma a_{i j} \delta_{j}$ e $\left(a_{i j}\right)$ é uma matriz $m \times m$ invertível com $a_{i j} \in F$. De fato, se substituirmos $\delta_{1}, \ldots, \delta_{m}$ por $\beta_{1}, \ldots, \beta_{m}$ usando uma transformação $F$-linear $\left(a_{i j}\right)$, podemos também substituir os geradores livres $\varepsilon_{1}, \ldots, \varepsilon_{m}$ de $S^{m}$ por $\zeta_{1}, \ldots, \zeta_{m}$ onde $\zeta_{i}=\Sigma b_{i j} \varepsilon_{j},\left(b_{i j}\right)=\left(a_{i j}\right)^{-1}$.

Seja $\operatorname{ord}\left(\delta_{i}\right)$ a ordem de $\delta_{i}$. Podemos supor que as ordens são decrescentes, ou seja, $\operatorname{ord}\left(\delta_{1}\right) \geqq \operatorname{ord}\left(\delta_{2}\right) \geqq \cdots \geqq \operatorname{ord}\left(\delta_{m}\right)$. Então, existe um inteiro positivo $\omega$ e algum inteiro $l, 1 \leq l \leq m$ tal que $\omega=\operatorname{ord}\left(\delta_{1}\right)=\cdots=\operatorname{ord}\left(\delta_{l}\right)$ e $\operatorname{ord}\left(\delta_{i}\right)<\omega$ se $i>l$.

Se $1 \leq i \leq l$ podemos escrever $\delta_{i}=r_{i}+p_{i}(x) \partial^{\omega}$, onde $\operatorname{ord}\left(r_{i}\right)<\omega$ e $p_{i}(x) \in F[[x]]$. Podemos supor que $\operatorname{val}\left(p_{1}\right) \leq \operatorname{val}\left(p_{2}\right) \leq \ldots \leq \operatorname{val}\left(p_{l}\right)$, onde $\operatorname{val}\left(p_{i}\right)$ é a valorização usual da série de potências $p_{i}$. Se $\operatorname{val}\left(p_{1}\right)=\operatorname{val}\left(p_{2}\right)=\mu$, então existe algum $t \in F$ tal que $\operatorname{val}\left(p_{2}-t p_{1}\right)>\mu$. Substituímos $\delta_{2}$ por $\delta_{2}-t \delta_{1}$ enquanto $\delta_{1}, \delta_{3}, \ldots, \delta_{m}$ não mudam. Então, depois de algumas transformações $F$-lineares, podemos assumir que $\operatorname{val}\left(p_{1}\right)<$ $\operatorname{val}\left(p_{2}\right)<\cdots<\operatorname{val}\left(p_{l}\right)$. Com essas normalizações em mão começamos a provar que $\varepsilon_{1} \in M$.

Seja $k=\operatorname{ord}(\alpha)$. Como $S=T((x))\langle\partial\rangle$ podemos supor que $\alpha=\alpha_{0}+\partial^{k}$ onde $\operatorname{ord}\left(\alpha_{0}\right)<k$. Se $1 \leq i \leq l$ temos que $\alpha \delta_{i}=p_{i}(x) \partial^{k+\omega}+\psi_{i}$ onde $\operatorname{ord}\left(\psi_{i}\right)<k+\omega$. Se $l<i \leq m$ então $\operatorname{ord}\left(\alpha \delta_{i}\right)<k+\omega$.

Dado $g \in S$ ponhamos $g_{1}=[x, g]=x g-g x$ o comutador de $x$ e $g$. Indutivamente, seja $g_{\nu+1}=\left[x, g_{\nu}\right]$. O elemento $g_{\nu}$ é chamado o $\nu$-comutador de $x$ e $g$. $\mathrm{O} \nu$-comutador de $x$ e $\partial^{\nu}$ é $(-1)^{\nu} \nu$ ! para todos os inteiros positivos $\nu$, enquanto o $\nu$-comutador de $x$ e $\partial^{s}$ é zero se $s<\nu$. 
Se aplicarmos isto aos elementos $\alpha \delta_{1}, \ldots, \alpha \delta_{m}$ vemos que o $(k+\omega)$ - comutador de $x$ e $\alpha \delta_{i}$ é $(-1)^{k+\omega}(k+\omega) ! p_{i}(x)$ para todo $1 \leq i \leq l$, enquanto eles são zero se $l<i \leq m$.

A definição de $M$ implica que $M$ é estável sob o $\nu$-comutador com $x$, ou seja se $m \in M$, então o $\nu$-comutador de $x$ e $m$ permanece em $M$, para todos os inteiros positivos $\nu$. De fato, note que se $f \in \mathbb{Z}[[x]]\langle\partial\rangle$, então $[x, \alpha \delta f]=x(\alpha \delta f)-\alpha \delta(f x)$.

Para $f=1 \in \mathbb{Z}[[x]]\langle\partial\rangle$ temos que $a=\alpha \delta_{1} \varepsilon_{1}+\cdots+\alpha \delta_{m} \varepsilon_{m} \in M$. Se $v_{1}$ é o $(k+\omega)-$ comutador de $a$ e $x$, dividido por $(-1)^{k+\omega}(k+\omega)$ !, então $v_{1} \in M$ e

$$
v_{1}=p_{1}(x) \varepsilon_{1}+\cdots+p_{l}(x) \varepsilon_{l}
$$

Agora, se $h \in S$, seja $h_{1}=[\partial, h]=\partial h-h \partial$ o comutador $\partial$ com $h$. Indutivamente definimos $h_{\nu+1}$ por $h_{\nu+1}=\left[\partial, h_{\nu}\right]$. O elemento $h_{\nu}$ é chamado o $\nu$-comutador de $\partial \mathrm{e}$ $h$. Observemos que $M$ é estável sob o $\nu$-comutador com $\partial$ para todos os inteiros positivos $\nu$. De fato, note que se $f \in \mathbb{Z}[[x]]\langle\partial\rangle$, então $[\partial, \alpha \delta f]=\partial(\alpha \delta f)-\alpha \delta(f \partial)$.

Como $v_{1} \in M$ temos que se $v_{1}^{(\mu)}$ é o $\mu$-comutador de $\partial$ e $v_{1}$, onde $\mu=\operatorname{val}\left(p_{1}(x)\right)<$ $\cdots<\operatorname{val}\left(p_{l}(x)\right)$, então $v_{1}^{(\mu)} \in M \mathrm{e}$

$$
v_{1}^{(\mu)}=p_{1}^{(\mu)}(x) \varepsilon_{1}+p_{2}^{(\mu)}(x) \varepsilon_{2}+\cdots+p_{l}^{(\mu)}(x) \varepsilon_{l} \in M
$$

onde $p^{(\mu)}(x)$ denota a $\mu$-derivada usual da série de potências $p(x)$. Note que $u(x):=$ $p_{1}^{(\mu)}(x)$ é uma unidade de $F[[x]]$ e $\operatorname{val}\left(p_{j}^{(\mu)}(x)\right)=\operatorname{val}\left(p_{j}(x)\right)-\mu>0, j=2, \cdots, l$.

Definimos agora $v_{2}:=v_{1}-p_{1}(x)(u(x))^{-1} v_{1}^{(\mu)}$. Então $v_{2} \in M \mathrm{e}$

$$
v_{2}=q_{2}(x) \varepsilon_{2}+\cdots+q_{l}(x) \varepsilon_{l}
$$

onde $q_{j}(x)=p_{j}(x)-p_{1}(x)(u(x))^{-1} p_{j}^{(\mu)}(x), j=2, \ldots, l$. Se para cada $2 \leq j \leq l$ escrevemos $p_{j}(x)=x^{v_{j}} u_{j}(x)$, onde $v_{j}=\operatorname{val}\left(p_{j}(x)\right)$ e $u_{j}(x)$ é uma unidade de $F[[x]]$, então

$q_{j}(x)=a_{j} x^{v a l\left(p_{j}(x)\right)}+\cdots$, onde $a_{j}:=\left(1-\frac{\left(v_{j}\right)\left(v_{j}-1\right) \cdots\left(v_{j}-(\mu-1)\right)}{\mu !}\right) u_{j}(0)$ é diferente de zero, pois $v_{j}>\mu$ e $u_{j}(0) \neq 0$. Então $q_{j}(x)$ é diferente de zero e mais ainda $\operatorname{val}\left(q_{j}(x)\right)=\operatorname{val}\left(p_{j}(x)\right)$, para todo $j=2, \ldots, l$. Portanto $\operatorname{val}\left(q_{2}\right)<\operatorname{val}\left(q_{3}\right)<\cdots<\operatorname{val}\left(q_{l}\right)$.

Agora, repetimos o argumento anterior usando o comutador de $\partial$ e $v_{2}$ e então temos que $v_{3}=r_{3}(x) \varepsilon_{3}+\cdots+r_{l}(x) \varepsilon_{l} \in M$. Procedendo desta maneira, finalmente temos que $v_{l}(x)=\widetilde{u}(x) \varepsilon_{l} \in M$, onde $\widetilde{u}(x)$ é uma unidade de $F[[x]]$. Consequentemente $\varepsilon_{l} \in M$.

Se $l=1$, mostramos que $\varepsilon_{1} \in M$. Se $l>1$, como $v_{l-1} \in M$, temos que $\varepsilon_{l-1} \in M$. Seguindo para trás temos que $\varepsilon_{1} \in M$.

Se agora restringimos nossa atenção à $(m-1)$-upla $\delta_{2}, \ldots, \delta_{m}$ e ao $S$-módulo $S^{m-1}=$ $S \varepsilon_{2}+\cdots+S \varepsilon_{m}$, a proposição segue-se por indução sobre $m$. 
Módulos Livres sobre $S=T((x))\left\langle\partial_{x}\right\rangle$ e sobre $D=F[[x]]\left\langle\partial_{x}\right\rangle$

Antes da próxima proposição faremos um resultado técnico.

Lema 3.7. Seja $S$ um domínio de Ore que não é Artiniano. Seja $S^{m}=S \varepsilon_{1}+\cdots+S \varepsilon_{m} u m$ $S$-módulo livre de posto $m$ com base $\varepsilon_{1}, \ldots, \varepsilon_{m}$. Seja $M \subset S^{m}$ um sobmódulo de co-comprimento finito. Então existe $\beta \in S, \beta \neq 0$, tal que $\beta \varepsilon_{i} \in M$, para todo $i=1, \ldots, m$.

Prova: Seja $\bar{\lambda} \in S^{m} / M, \bar{\lambda} \neq \overline{0}, \lambda \in S^{m}$. Temos $S / \operatorname{ann}(\bar{\lambda}) \simeq S \bar{\lambda} \subset S^{m} / M$. Se $\operatorname{ann}(\bar{\lambda})=(0)$, então $S \simeq S \bar{\lambda}$ que é um S-módulo Artiniano pois $M$ tem co-comprimento finito em $S^{m}$. Logo $S$ seria Artiniano. Assim $\operatorname{ann}(\bar{\lambda}) \neq(0)$.

Como $S^{(m)} / M=S \overline{\varepsilon_{1}}+\cdots+S \overline{\varepsilon_{m}}$ com $\operatorname{ann}\left(\overline{\varepsilon_{i}}\right) \neq 0$, existe $q_{j} \in S, q_{j} \neq 0$ tal que $q_{j} \varepsilon_{j} \in M$. Já que $S$ é um domínio de Ore, existe $\beta=\lambda_{j} q_{j} \in S q_{j}, \beta \neq 0, j=1, \ldots, m$. $\operatorname{Logo} \beta \varepsilon_{j}=\left(\lambda_{j} q_{j}\right) \varepsilon_{j}=\lambda_{j}\left(q_{j} \varepsilon_{j}\right) \in M$.

Proposição 3.8. (i) Sejam $\delta_{1}, \ldots, \delta_{m}$ elementos diferentes de zero de $D=F[[x]]\left\langle\partial_{x}\right\rangle \subset S e$ seja $M$ um $S$-submódulo de $S^{m}$ de co-comprimento finito. Se $\alpha \in S, \alpha \neq 0$, então existe algum $f \in \mathbb{Z}[[x]]\left\langle\partial_{x}\right\rangle$ tal que

$$
S^{m}=M+S\left(\alpha \delta_{1} f \varepsilon_{1}+\cdots+\alpha \delta_{m} f \varepsilon_{m}\right) .
$$

(ii) Sejam $\delta_{1}, \ldots, \delta_{m}$ elementos diferentes de zero de $D=F[[x]]\left\langle\partial_{x}\right\rangle$ e seja $M$ um $D$-submódulo de $D^{m}$ de co-comprimento finito. Se $\alpha \in D, \alpha \neq 0$, então existe algum $f \in \mathbb{Z}[[x]]\left\langle\partial_{x}\right\rangle$ tal que

$$
D^{m}=M+D\left(\alpha \delta_{1} f \varepsilon_{1}+\cdots+\alpha \delta_{m} f \varepsilon_{m}\right) .
$$

Prova: Vamos mostrar $(i)$. A prova de (ii) é análoga.

Usaremos indução sobre o comprimento do $S$-módulo $S^{(m)} / M$.

Podemos assumir que $M \neq S^{(m)}$. Segue-se da proposição 2.5 que existe $f_{0} \in \mathbb{Z}[[x]]$ tal que

$$
\alpha \delta_{1} f_{0} \varepsilon_{1}+\cdots+\alpha \delta_{m} f_{0} \varepsilon_{m} \notin M
$$

Consideremos agora um $S$-submódulo $M^{\prime}$ de $S^{m}$ tal que $M \subset M^{\prime} \subset M+S\left(\sum_{j} \alpha \delta_{j} f_{0} \varepsilon_{j}\right)$ e tal que $M / M^{\prime}$ seja irredutível. Note que $M^{\prime}$ existe pois o comprimento de $S^{m} / M$ é finito.

Pelo lema anterior, existe $\beta \in S, \beta \neq 0$ tal que $\beta \varepsilon_{i} \in M, i=1, \ldots, m$. Logo $S^{m} \beta \subset M$. Como $S$ é um domínio de Ore, existe $t \in S, t \neq 0$ tal que $t \alpha \delta_{j} f_{0} \in S \beta, j=1, \ldots, m$.

A hipótese de indução aplicada a $M^{\prime}$ e $\alpha^{\prime}=t \alpha$ garante a existência de $f_{1} \in \mathbb{Z}[[x]]$ tal que $S^{m}=M^{\prime}+S\left(\sum_{j} t \alpha \delta_{j} f_{1} \varepsilon_{j}\right)$. 
Seja $N:=M+S\left(\sum_{j} \alpha \delta_{j} f_{1} \varepsilon_{j}\right)$. Se $N=S^{m}$, a prova acabou pois $f_{1}$ cumpre as condições requeridas. Suponhamos então que $N \neq S^{m}$. Neste caso provaremos que $S^{m}=M+\left(\sum_{j} \alpha \delta_{j}\left(f_{0}+f_{1}\right) \varepsilon_{j}\right)$ e por tanto $f:=f_{0}+f_{1}$ é o elemento procurado.

Afirmação: Se $N \neq S^{m}$ então $N=N_{0}$, onde $N_{0}:=M+S\left(\sum_{j} t \alpha \delta_{j} f_{1} \varepsilon_{j}\right)$. Com efeito, desde que $S^{m}=M^{\prime}+P$, onde $P=S\left(\sum_{j} t \alpha \delta_{j} f_{1} \varepsilon_{j}\right)$, temos que

$$
S^{m} / N_{0}=\left(M^{\prime}+P\right) /(M+P) \simeq M^{\prime} /\left(M+M^{\prime} \cap P\right) \subset M^{\prime} / M .
$$

Como $M^{\prime} / M$ é irredutível, $S^{m} / N_{0}$ é zero ou irredutível. Mas $N_{0} \subset N$ pois $M \subset N$ e $\sum_{j} t \alpha \delta_{j} f_{1} \varepsilon_{j}=t\left(\sum_{j} \alpha \delta_{j} f_{1} \varepsilon_{j}\right) \in N$. Logo $N_{0} \neq S^{m}$ e portanto $S^{m} / N_{0}$ é irredutível. Como $N_{0} \subset N \subset S^{m}$ e $N \neq S^{m}$, então $N=N_{0}$, o que prova a afirmação.

Seja agora $N_{1}:=M+S\left(\sum_{j} \alpha \delta_{j}\left(f_{0}+f_{1}\right) \varepsilon_{j}\right)$. Vamos mostrar que $N_{1}=S^{m}$. Pela escolha de $t, t \alpha \delta_{j} f_{0} \varepsilon_{j} \in S \beta, j=1, \ldots, m$ e como $S^{m} \beta \subset M$, então $\sum_{j} t \alpha \delta_{j} f_{0} \varepsilon_{j} \in M$. Mas $M \subset N_{1}$ e $\sum_{j} \alpha \delta_{j}\left(f_{0}+f_{1}\right) \varepsilon_{j} \in N_{1}$. Logo $\sum_{j} \alpha \delta_{j} f_{1} \varepsilon_{j} \in N_{1}$. Além disso, pela escolha de $\left.M^{\prime}, M^{\prime} \subset M+S\left(\sum_{j} \alpha \delta_{j} f_{0} \varepsilon_{j}\right)\right) \subset N_{1}$.

Finalmente, $t\left(\sum_{j} \alpha \delta_{j} f_{1} \varepsilon_{j}\right) \in S\left(\sum_{j} \alpha \delta_{j} f_{1} \varepsilon_{j}\right) \subset N_{1}$. Consequentemente,

$$
S^{m}=M^{\prime}+S\left(\sum_{j} t \alpha \delta_{j} f_{1} \varepsilon_{j}\right) \subset N_{1}
$$

Corolário 3.9. (i) Seja $\rho \in S, \rho \neq 0$ e sejam $\delta_{1}, \ldots, \delta_{m}$ elementos diferentes de zero em $F[[x]]\left\langle\partial_{x}\right\rangle \subset S$. Então existe algum $f \in \mathbb{Z}[[x]]\left\langle\partial_{x}\right\rangle$ tal que

$$
S^{m}=S^{m} \rho+S\left(\rho \delta_{1} f \varepsilon_{1}+\cdots+\rho \delta_{m} f \varepsilon_{m}\right),
$$

onde $S^{m} \rho$ é o $S$-submódulo de $S^{m}$ gerado por $\rho \varepsilon_{1}, \ldots, \rho \varepsilon_{m}$.

(ii) Seja $\rho \in D, \rho \neq 0$ e sejam $\delta_{1}, \ldots, \delta_{m}$ elementos diferentes de zero de $D$. Então existe algum $f \in \mathbb{Z}[[x]]\left\langle\partial_{x}\right\rangle$ tal que

$$
D^{m}=D^{m} \rho+D\left(\rho \delta_{1} f \varepsilon_{1}+\cdots+\rho \delta_{m} f \varepsilon_{m}\right)
$$

onde $D^{m} \rho$ é o D-submódulo de $D^{m}$ gerado por $\rho \varepsilon_{1}, \ldots, \rho \varepsilon_{m}$.

Prova: (i): Pela proposição 3.4, o comprimento do $S$-módulo $S / S \rho$ é finito e portanto $S^{m} \rho$ tem co-comprimento finito em $S^{m}$. O resultado segue-se então se aplicarmos a proposição $3.8(i) \operatorname{com} \alpha=\rho$ e $M=S^{m} \rho$. 
(ii): Pela proposição 3.5, o comprimento do $D$-módulo $D / D \rho$ é finito e portanto $D^{m} \rho$ tem co-comprimento finito em $D^{m}$. O resultado segue-se se aplicarmos a proposição $3.8(i i) \operatorname{com} \alpha=\rho$ e $M=D^{m} \rho$.

Proposição 3.10. (i) Sejam $\delta_{1}, \ldots, \delta_{m}$ elementos diferentes de zero de $F[[x]]\left\langle\partial_{x}\right\rangle \subset S$ e seja $\rho \in S, \rho \neq 0$. Consideremos o $S$-módulo livre $S^{m+1}=S \varepsilon_{0}+S \varepsilon_{1}+\cdots+S \varepsilon_{m}$ com base $\varepsilon_{0}, \varepsilon_{1}, \ldots, \varepsilon_{m}$. Então existe algum $f \in \mathbb{Z}[[x]]\left\langle\partial_{x}\right\rangle$ tal que

$$
S^{m+1}=S^{m+1} \rho+S\left(\varepsilon_{0}+\delta_{1} f \varepsilon_{1}+\cdots+\delta_{1} f \varepsilon_{m}\right)
$$

onde $S^{m+1} \rho$ é o $S$-submódulo de $S^{m+1}$ gerado por $\rho \varepsilon_{0}, \ldots, \rho \varepsilon_{m}$.

(ii) Sejam $\delta_{1}, \ldots, \delta_{m}$ elementos diferentes de zero de $D=e$ seja $\rho \in D, \rho \neq 0$. Consideremos o D-módulo livre $D^{m+1}=D \varepsilon_{0}+D \varepsilon_{1}+\cdots+D \varepsilon_{m}$ com base $\varepsilon_{0}, \cdots, \varepsilon_{m}$. Então existe algum $f \in \mathbb{Z}[[x]]\left\langle\partial_{x}\right\rangle$ tal que

$$
D^{m+1}=D^{m+1} \rho+D\left(\varepsilon_{0}+\delta_{1} f \varepsilon_{1}+\cdots+\delta_{1} f \varepsilon_{m}\right),
$$

onde $D^{m+1} \rho$ é o D-submódulo de $D^{m+1}$ gerado por $\rho \varepsilon_{0}, \ldots, \rho \varepsilon_{m}$.

Prova: Vamos mostrar $(i)$. A prova de $(i i)$ é análoga.

O corolário anterior garante a existência de $f \in \mathbb{Z}[[x]]\left\langle\partial_{x}\right\rangle$ tal que

$$
S^{m}=S^{m} \rho+S\left(\rho \delta_{1} f \varepsilon_{1}+\cdots+\rho \delta_{m} f \varepsilon_{m}\right)
$$

Logo, se $v \in S^{m+1}$, então existem $\alpha_{0} \in S$ e $w \in S^{m}$ tais que $v=\alpha_{0} \varepsilon_{0}+w$. Pela igualdade anterior, $w=\beta_{1} \rho \varepsilon_{1}+\cdots+\beta_{m} \rho \varepsilon_{m}+\lambda\left(\rho \delta_{1} f \varepsilon_{1}+\cdots+\rho \delta_{m} f \varepsilon_{m}\right)$, para alguns $\beta_{1}, \ldots, \beta_{m}, \lambda \in S$. Por outro lado, pelo algoritmo da divisão em $S$, podemos escrever $\alpha_{0}=\beta_{0} \rho+\theta$, para alguns $\beta_{0}, \theta \in S$. Então

$v=\left(\beta_{0} \rho \varepsilon_{0}+\beta_{1} \rho \varepsilon_{1}+\cdots+\beta_{m} \rho \varepsilon_{m}\right)+\lambda \rho\left(\varepsilon_{0}+\delta_{1} f \varepsilon_{1}+\cdots+\delta_{m} f \varepsilon_{m}\right)+(\theta-\lambda \rho) \varepsilon_{0}$. Logo

$$
S^{m+1}=S^{m+1} \rho+S\left(\varepsilon_{0}+\rho \delta_{1} f \varepsilon_{1}+\cdots+\rho \delta_{m} f \varepsilon_{m}\right)+S \varepsilon_{0} .
$$

Seja $J:=S^{m+1} \rho+S\left(\varepsilon_{0}+\delta_{1} f \varepsilon_{1}+\cdots+\delta_{m} f \varepsilon_{m}\right)$. Então

$$
J \supset S^{m+1} \rho+S \rho\left(\varepsilon_{0}+\delta_{1} f \varepsilon_{1}+\cdots+\delta_{m} f \varepsilon_{m}\right) \supset S^{m+1} \rho+S \rho\left(\delta_{1} f \varepsilon_{1}+\cdots+\delta_{m} f \varepsilon_{m}\right) .
$$

De fato, a primeira inclusão é clara. Para mostrar a segunda inclusão notemos que se $v \in S \rho\left(\delta_{1} f \varepsilon_{1}+\cdots+\delta_{m} f \varepsilon_{m}\right)$, então $v=\lambda \rho\left(\delta_{1} f \varepsilon_{1}+\cdots+\delta_{m} f \varepsilon_{m}\right)$, para algum $\lambda \in S$. Logo 
$v=(-\lambda \rho) \varepsilon_{0}+\lambda \rho\left(\varepsilon_{0}+\delta_{1} f \varepsilon_{1}+\cdots+\delta_{m} f \varepsilon_{m}\right) \in S^{m+1} \rho+S \rho\left(\varepsilon_{0}+\delta_{1} f \varepsilon_{1}+\cdots+\delta_{m} f \varepsilon_{m}\right)$.

Por outro lado,

$$
S^{m+1} \rho+S \rho\left(\delta_{1} f \varepsilon_{1}+\cdots+\delta_{m} f \varepsilon_{m}\right) \supset S \rho \varepsilon_{0}+S^{m} .
$$

Com efeito, seja $v \in S \rho \varepsilon_{0}+S^{m}$, então $v=\lambda_{0} \rho \varepsilon_{0}+w$, onde $\lambda_{0} \in S$ e $w \in S^{m}$. Logo, por $(*)$, existem $\lambda_{1}, \ldots, \lambda_{m}, \beta \in S$ tais que $w=\lambda_{1} \rho \varepsilon_{1}+\cdots+\lambda_{m} \rho \varepsilon_{m}+\beta\left(\rho \delta_{1} f \varepsilon_{1}+\cdots+\rho \delta_{m} f \varepsilon_{m}\right)$. Então $v=\left(\lambda_{0} \rho \varepsilon_{0}+\lambda_{1} \rho \varepsilon_{1}+\cdots+\lambda_{m} \rho \varepsilon_{m}\right)+\beta \rho\left(\delta_{1} f \varepsilon_{1}+\cdots+\delta_{m} f \varepsilon_{m}\right) \in S^{m+1} \rho+S \rho\left(\delta_{1} f \varepsilon_{1}+\right.$ $\left.\cdots+\delta_{m} f \varepsilon_{m}\right) \in S^{m+1} \rho+S \rho\left(\delta_{1} f \varepsilon_{1}+\cdots+\delta_{m} f \varepsilon_{m}\right)$.

Mas, $\left(\delta_{1} f \varepsilon_{1}+\cdots+\delta_{m} f \varepsilon_{m}\right) \in S \rho \varepsilon_{0}+S^{m}$. Então das inclusões anteriores temos que $\left(\delta_{1} f \varepsilon_{1}+\cdots+\delta_{m} f \varepsilon_{m}\right) \in J$. Isto implica que $\varepsilon_{0} \in J$.

Usando agora $(* *)$, concluímos que $J=S^{m+1}$, o que demonstra a proposição. 


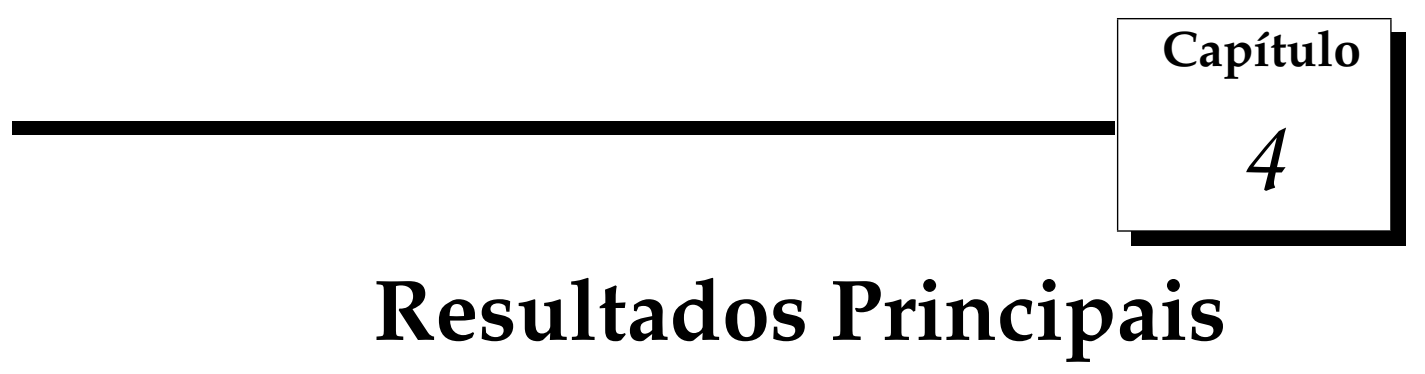

Seja $K$ um corpo de característica zero. Neste capítulo provaremos que todo ideal à esquerda do anel $E_{n}(K):=K\left(\left(x_{1}, \ldots, x_{n}\right)\right)\left\langle\partial_{1}, \ldots, \partial_{n}\right\rangle$ é dois gerado. Mostraremos também que todo ideal à esquerda do anel $S_{n-1}=K\left(\left(x_{1}, \ldots, x_{n-1}\right)\right)\left[\left[x_{n}\right]\right]\left\langle\partial_{1}, \ldots, \partial_{n}\right\rangle$ é dois gerado. Como corolário, para $n=1$, obtemos outra prova do fato que todo ideal à esquerda do anel $D_{1}(K)=S_{0}(K)$ é dois gerado. Os resultados mais importantes são os teoremas $4.8 \mathrm{e} 4.13$.

Para cada $0 \leq r \leq n$, os anéis $D_{r}(K)=K\left[\left[x_{1}, \ldots, x_{r}\right]\right]\left\langle\partial_{1}, \ldots, \partial_{r}\right\rangle$ serão denotados simplesmente por $D_{r}$.

\subsection{Os anéis $B_{r}(K)$}

Definição 4.1. Seja $r$ um inteiro positivo tal que $1 \leq r \leq n-1$ e seja q um elemento diferente de zero do anel $D_{r}\left[\left[x_{r+1}, \ldots, x_{n}\right]\right]$. Um tal $q$ se escreve unicamente na forma $q=\sum_{\nu} q_{\nu} x^{\nu}$, onde $\nu=\left(\nu_{r+1}, \ldots, \nu_{n}\right) \in \mathbb{N}^{n-r}, x=\left(x_{r+1}, \ldots, x_{n}\right)$ e $q_{\nu} \in D_{r}$. Dado $i=1, \ldots, r$, dizemos que $q$ tem ordem finita em $\partial_{i}$, se existe algum $m \in \mathbb{N}$ tal que ord $\partial_{i} q_{\nu} \leq m$, para todo $\nu$.

O conjunto dos elementos do anel $D_{r}\left[\left[x_{r+1}, \ldots, x_{n}\right]\right]$ que têm ordem finita em $\partial_{i}$, para todo $1 \leq i \leq r$, será denotado por $B_{r}(K)$ ou simplesmente $B_{r}$. Em outras palavras, para $1 \leq r \leq n-1$,

$B_{r}(K):=\left\{q \in D_{r}\left[\left[x_{r+1}, \ldots, x_{n}\right]\right]\right.$ tal que $q$ têm ordem finita $\partial_{i}$, para todo $\left.1 \leq i \leq r\right\}$

Por definição $B_{0}(K):=K\left[\left[x_{1}, \ldots, x_{n}\right]\right]$.

Proposição 4.2. Seja $r$ um inteiro positivo tal que $1 \leq r \leq n-1$. Então $B_{r}(K)=$ 
$K\left[\left[x_{1}, \ldots, x_{n}\right]\right]\left\langle\partial_{1}, \ldots, \partial_{r}\right\rangle$ e portanto $B_{r}(K)$ é um subanel do anel $D_{n}(K)=K\left[\left[x_{1}, \ldots, x_{n}\right]\right]\left\langle\partial_{1}, \ldots, \partial_{n}\right\rangle$. Mas ainda, $B_{r}(K)$ é um dominio de Ore.

Prova: Seja $q=\sum_{\nu} q_{\nu} x^{\nu} \in B_{r}(K)$ onde $\nu=\left(\nu_{r+1}, \ldots, \nu_{n}\right) \in \mathbb{N}^{n-r}, x=\left(x_{r+1}, \ldots, x_{n}\right)$ e $q_{\nu} \in D_{r}$, para todo $\nu$. Por definição, existe um natural $m \in \mathbb{N}$ tal que para cada $\nu$ nos podemos escrever $q_{\nu}=\sum_{\beta} q_{\nu, \beta} \partial^{\beta}$ como uma soma finita, onde $\beta=\left(\beta_{1}, \ldots, \beta_{r}\right)$, $q_{\nu, \beta} \in K\left[\left[x_{1}, \ldots, x_{r}\right]\right], \partial^{\beta}=\partial_{1}^{\beta_{1}} \cdots \partial_{r}^{\beta_{r}}$ e $|\beta|=\beta_{1}+\cdots+\beta_{r} \leq m$. Então, para cada $\nu$, temos $q_{\nu} x^{\nu}=\left(\sum_{\beta} q_{\nu, \beta} \partial^{\beta}\right) x^{\nu}=\sum_{\beta} q_{\nu, \beta} x^{\nu} \partial^{\beta}$, pois os $x^{\nu}$ comutam com os $\partial^{\beta}$. Agora notemos que para cada $\beta \operatorname{com}|\beta| \leq m$, a soma $p_{\beta}:=\sum_{\nu} q_{\nu, \beta} x^{\nu}$ é um elemento de $K\left[\left[x_{1}, \ldots, x_{r}\right]\right]\left[\left[x_{r+1}, \ldots, x_{n}\right]\right]=K\left[\left[x_{1}, \ldots, x_{n}\right]\right]$. Logo,

$q=\sum_{\nu} q_{\nu} x^{\nu}=\sum_{\nu} \sum_{\beta} q_{\nu, \beta} x^{\nu} \partial^{\beta}=\sum_{\beta}\left(\sum_{\nu} q_{\nu, \beta} x^{\nu}\right) \partial^{\beta}=\sum_{\beta} p_{\beta} \partial^{\beta} \in K\left[\left[x_{1}, \ldots, x_{n}\right]\right]\left\langle\partial_{1}, \ldots, \partial_{r}\right\rangle$.

Por outro lado, é claro que $K\left[\left[x_{1}, \ldots, x_{n}\right]\right]\left\langle\partial_{1}, \ldots, \partial_{r}\right\rangle$ está contido em $B_{r}(K)$. Portanto $B_{r}(K)=K\left[\left[x_{1}, \ldots, x_{n}\right]\right]\left\langle\partial_{1}, \ldots, \partial_{r}\right\rangle$ que é um subanel de $D_{n}(K)$.

O anel $K\left[\left[x_{1}, \ldots, x_{n}\right]\right]\left\langle\partial_{1}, \ldots, \partial_{r}\right\rangle$ possui uma filtração canônica pela ordem dada por

$\Sigma_{m}:=\left\{\sum_{\beta} p_{\beta}(x) \partial^{\beta},|\beta|=\beta_{1}+\cdots+\beta_{r} \leq m\right\}, m \geq 0$. O anel graduado associado à esta filtração é isomorfo ao anel de polinômios $K\left[\left[y_{1}, \ldots, y_{n}\right]\right]\left[z_{1}, \ldots, z_{r}\right]$. Como o último anel é Noetheriano, concluimos que $K\left[\left[x_{1}, \ldots, x_{n}\right]\right]\left\langle\partial_{1}, \ldots, \partial_{r}\right\rangle$ é um anel Noetheriano. Logo $B_{r}(K)$ é um domínio Noetheriano e em consequência é um domínio de Ore.

Proposição 4.3. Seja $r$ um inteiro não negativo tal que $0 \leq r \leq n-1, q \in B_{r}(K), q \neq 0$. Sejam $a_{1}, \ldots, a_{l}$ elementos de $D_{n}$. Então existe $\rho \in B_{r}(K), \rho \neq 0$, tal que $\rho a_{j} \in D_{n} q$, para cada $j=1, \ldots, l$.

Prova: Cada $a_{j}$ pode ser escrito como uma soma finita $\sum_{\alpha} p_{j \alpha} \partial^{\alpha}$ onde cada $p_{j \alpha}$ é um elemento de $B_{r}(K)=K\left[\left[x_{1}, \ldots, x_{n}\right]\right]\left\langle\partial_{1}, \ldots, \partial_{r}\right\rangle$ e $\partial^{\alpha}=\partial_{r+1}^{\alpha_{r+1}} \ldots \partial_{n}^{\alpha_{n}}$. Escolhemos um inteiro positivo $w$ suficientemente grande tal que $p_{j \alpha}=0$ para todo $\alpha$ tal que $|\alpha| \geq w$ e para todo $j=1, \ldots, l$.

Como $B_{r}(K)$ é um domínio de Ore e $q^{w} \neq 0 B_{r}(K)$, existe $\rho^{\prime} \in B_{r}, \rho_{1} \neq 0$, tal que $\rho^{\prime} p_{j \alpha} \in B_{r} q^{w}$ para todo $j$ e todo $\alpha$.

Logo $\rho^{\prime} p_{j \alpha}=s_{j \alpha} q^{w}$ para algum $s_{j \alpha} \in B_{r}$. Temos que $\rho^{\prime} a_{j}=\sum_{\mid \alpha<w} p_{j \alpha} \partial^{\alpha}=$ $\sum_{|\alpha|<w} s_{j \alpha} q^{w} \partial^{\alpha}$. Por outro lado, para cada $|\alpha|<w$, podemos escrever $q^{w} \partial^{\alpha}=t_{\alpha} q+q q_{\alpha}$, para alguns $t_{\alpha} \in D_{n}$ e $q_{\alpha} \in B_{r}$. Para cada $q_{\alpha} \neq 0$, existem $0 \neq r_{\alpha}, s_{\alpha} \in B_{r}$ tais que $r_{\alpha} q_{\alpha}=s_{\alpha} q$, pois $B_{r}(K)$ é um domínio de Ore.

Se $C_{r}(K)$ denota o anel de frações de $B_{r}(K)$, então em $C_{r}(K)$ temos a relação $q_{\alpha}=$ 
$r_{\alpha}^{-} 1 s_{\alpha} q$. Logo em $C_{n}(K)$, o anel de frações de $B_{n}(K)=D_{n}(K)$, temos

$\rho^{\prime} a_{j}=\sum_{|\alpha|<w}\left(s_{j \alpha} t_{\alpha}\right) q+\sum_{|\alpha|<w, q_{\alpha} \neq 0}\left(q r_{\alpha}^{-1} s_{\alpha}\right) q=\left(\sum_{|\alpha|<w} s_{j \alpha} t_{\alpha}\right) q+\left(\sum_{|\alpha|<w, q_{\alpha} \neq 0} q r_{\alpha}^{-1} s_{\alpha}\right) q=\beta_{j} q+\gamma_{j} q$, onde $\beta_{j}:=\left(\sum_{|\alpha|<w} s_{j \alpha} t_{\alpha}\right) \in D_{n}$ e $\gamma_{j}:=\left(\sum_{|\alpha|<w, q_{\alpha} \neq 0} q r_{\alpha}^{-1} s_{\alpha}\right) \in C_{r}$.

Desde que $C_{r}(K)$ é o anel de frações de $B_{r}$, existem $\lambda_{j}, \mu_{j} \in B_{r}(K), \lambda_{j} \neq 0$ tais que $\gamma_{j}=\lambda_{j}^{-1} \mu_{j}$. Então $\rho^{\prime} a_{j}=\beta_{j} q+\lambda_{j}^{-1} \mu_{j} q$, para todo $j=1, \ldots, l$. Como $B_{r}(K)$ é um domínio de Ore, os $\lambda_{j}$ têm um múltiplo comum, ou seja, existe $\theta \in B_{r}, \theta \neq 0$ tal que $\theta \in B_{r}(K) \lambda_{j}$. Por tanto $\theta=\eta_{j} \lambda_{j}$, para alguns $\eta_{j} \in B_{r}(K)$. Logo $\theta \rho^{\prime} a_{j}=\theta \beta_{j} q+\eta_{j} \mu_{j} \in D_{n} q$ para todo $j=1, \ldots, l$. Então, se $\rho:=\theta \rho^{\prime}$, temos que $\rho \in B_{r}(K)$ e mais ainda $\rho a_{j} \in D_{n} q$ para todo $j=1, \ldots, l$.

\section{$4.2 \mathrm{O}$ anel $E_{n}(K)=K\left(\left(x_{1}, \ldots, x_{n}\right)\right)\left\langle\partial_{1}, \ldots, \partial_{n}\right\rangle$}

Seja $K$ um corpo de característica zero e $K\left(\left(x_{1}, . ., x_{n}\right)\right)$ o corpo de frações do anel de séries de potências $K\left[\left[x_{1}, \ldots, x_{n}\right]\right]$. Sobre $K\left(\left(x_{1}, . ., x_{n}\right)\right)$ temos as derivações usuais $\partial_{1}, \ldots, \partial_{n}$ que são operadores $K$-lineares.

Se $p$ é um elemento de $K\left(\left(x_{1}, . ., x_{n}\right)\right)$, então $p$ define un operador $K$-linear sobre $K\left(\left(x_{1}, . ., x_{n}\right)\right)$ que leva um elemento $q \in K\left(\left(x_{1}, . ., x_{n}\right)\right)$ no produto $p q$. Tal operador é chamado operador produto $p$.

Definição 4.4. O subanel dos $K$-operadores lineares de $K\left(\left(x_{1}, . ., x_{n}\right)\right)$ que é gerado pelas derivações usuais $\partial_{1}, \ldots, \partial_{n}$ e os operadores produto definidos por os elementos de $K\left(\left(x_{1}, . ., x_{n}\right)\right)$ é chamado anel de operadores diferenciais de $K\left(\left(x_{1}, . ., x_{n}\right)\right)$ e será denotado por $E_{n}(K)=K\left(\left(x_{1}, \ldots, x_{n}\right)\right)\left\langle\partial_{1}, \ldots, \partial_{n}\right\rangle$.

Queremos mostrar que todo ideal à esquerda do anel $E_{n}(K)=K\left(\left(x_{1}, \ldots, x_{n}\right)\right)\left\langle\partial_{1}, \ldots, \partial_{n}\right\rangle$ é dois gerado. Isso será consequência da seguinte proposição:

Proposição 4.5. Sejam $a, b$ e c elementos diferentes de zero de $D_{n}$. Para cada inteiro não negativo $r$ tal que $0 \leq r \leq n$, existem $q_{r} \in B_{r}, q_{r} \neq 0$ e $d_{r}, e_{r} \in D_{n}$ tais que

$$
q_{r} c \in D_{n}\left(a+d_{r} c\right)+D_{n}\left(b+e_{r} c\right) .
$$

Em particular, quando $r=0$, existem $q_{0} \in K\left[\left[x_{1}, \ldots, x_{n}\right]\right], q_{0} \neq 0$ e $d_{0}, e_{0} \in D_{n}$ tais que

$$
q_{0} c \in D_{n}\left(a+d_{0} c\right)+D_{n}\left(b+e_{0} c\right) .
$$


Para demonstrar a proposição acima necessitamos do seguinte resultado, cuja prova será adiada até a próxima seção (4.3).

Proposição 4.6. Seja $r$ um inteiro não negativo tal que $0 \leq r \leq n-1$ e seja q um elemento diferente de zero em $B_{r+1}$. Se u e $v$ são dois elementos de $D_{n}$ com $v \neq 0$, então existem $f$ em $D_{n}$ e $q_{r}$ em $B_{r}$ tal que

$$
q_{r} \in D_{n} q+D_{n}(u+v f) .
$$

Observação 4.7. Note que na proposição acima $q \in B_{r+1}$, enquanto $q_{r} \in B_{r}$. Assim, passando de $q_{r+1}$ a $q_{r}$ "eliminamos" a variável $\partial_{r+1}$. Eliminando cada variável $\partial_{i}$ por vez, chegamos a uma série $q_{0} \in K\left[\left[x_{1}, \ldots, x_{n}\right]\right]$.

\section{Prova da Proposição 4.5 .}

Prova: Como $D_{n}$ é um domínio de Ore à esquerda, $D_{n} a \cap D_{n} c \neq 0$. Portanto existe $q_{n} \in$ $D_{n}, q_{n} \neq 0$ tal que $q_{n} c \in D_{n} a$. Como $D_{n} a \subset D_{n} a+D_{n} b$, tomando $d_{n}=e_{n}=0$ vemos que a afirmação é verdadeira para $r=n$.

Para $0 \leq r \leq(n-1)$ a prova será feita por indução "para trás"em $r$. Suponhamos então que podemos achar $q_{r+1} \in B_{r+1}, q_{r+1} \neq 0$ e $d_{r+1}, e_{r+1} \in D_{n}$ tais que

$$
q_{r+1} c \in D_{n}\left(a+d_{r+1} c\right)+D_{n}\left(b+e_{r+1} c\right) .
$$

Podemos supor que $a+d_{r+1} c \neq 0$ e $b+e_{r+1} c \neq 0$, pois se por exemplo, $a+d_{r+1} c=0$, então $q_{r+1} c \in D_{n}\left(b+d_{r+1} c\right)$. Isto implica que $b+d_{r+1} c \neq 0$. Logo

$$
q_{r+1} c \in D_{n}\left(b+d_{r+1} c\right) \subset D_{n}\left(a+\left(d_{r+1}+1\right) c\right)+D_{n}\left(b+d_{r+1} c\right)
$$

onde $a+\left(d_{r+1}+1\right) c=c \neq 0 e b+d_{r+1} c \neq 0$.

Ponhamos $a^{\prime}=a+d_{r+1} c \neq 0 e b^{\prime}=b+e_{r+1} c \neq 0$. Se encontramos $q_{r} \in B_{r}, q_{r} \neq 0 e$ $d_{r}^{\prime}, e_{r}^{\prime} \in D_{n}$ tais que

$$
q_{r} c \in D_{n}\left(a^{\prime}+d_{r}^{\prime} c\right)+D_{n}\left(b^{\prime}+e_{r}^{\prime} c\right)
$$

então, tomando $d_{r}=d_{r+1}+d_{r}^{\prime}$ e $e_{r}=e_{r+1}+e_{r}^{\prime}$, temos que

$$
q_{r} c \in D_{n}\left(a+d_{r} c\right)+D_{n}\left(b+e_{r} c\right)
$$

Assim, sem perda de generalidade, podemos supor que $a^{\prime}=$ a e que $b^{\prime}=b$ desde o início.

Portanto podemos escrever

$$
q_{r+1} c=h_{1} a+h_{2} b
$$

para alguns $h_{1}, h_{2} \in D_{n}$. Podemos supor que $h_{1} h_{2} \neq 0$. De fato, se por exemplo, $h_{1}=0$, 
então $q_{r+1} c=h_{2}$ b e como $D_{n} a \cap D_{n} b \neq 0$, existem $s, t \in D_{n}$, st $\neq 0$ tais que $s a=t b$. Logo $q_{r+1} c=(-s) a+\left(h_{2}+t\right) b$. Se $\left(h_{2}+t\right) \neq 0$, então podemos tomar $(-s)$ em lugar de $h_{1} e$ $\left(h_{2}+t\right)$ no lugar de $h_{2}$. Se $h_{2}+t=0$, podemos escrever $q_{r+1} c=s a+\left(h_{2}-t\right) b$ e neste caso, $s$ pode substituir $h_{1} e\left(h_{2}-t\right)$ pode substituir $h_{2}$.

Além disso, como $D_{n}$ é um domínio de Ore à direita, $h_{1} D_{n} \cap h_{2} D_{n} \neq 0$ e portanto existem $g_{1}, g_{2}$ em $D_{n}$, ambos diferentes de zero, tais que

$$
h_{1} g_{1}=-h_{2} g_{2}
$$

Por outro lado, usando o fato que $D_{n}$ é um domínio de Ore à esquerda, existem s,t ambos diferentes de zero em $D_{n}$ tais que

$$
s q_{r+1} c=t b .
$$

Pela proposição 4.6 acima com $q=q_{r+1} \in B_{r+1}, u=0$ e $v=t g_{2}$, implica que existem $f \in D_{n}$ e $q_{r} \in B_{r}, q_{r} \neq 0$ tais que $q_{r} \in D_{n} q_{r+1}+D_{n}\left(t g_{2} f\right)$. Digamos

$$
q_{r}=p_{1} q_{r+1}+p_{2} t g_{2} f
$$

para alguns $p_{1}, p_{2}$ de $D_{n}$. Assim,

$$
q_{r} c=\left(p_{1} q_{r+1}+p_{2} t g_{2} f\right) c=p_{1} q_{r+1} c+p_{2} t g_{2} f c .
$$

Seja $\xi=p_{2} s q_{r+1} c=p_{2} t b$. Somando e subtraindo $\xi$ obtemos:

$$
\begin{gathered}
q_{r} c=\left(p_{1} q_{r+1} c-\xi\right)+\left(p_{2} t g_{2} f c+\xi\right)=\left(p_{1} q_{r+1} c-p_{2} s q_{r+1} c\right)+\left(p_{2} t g_{2} f c+p_{2} t b\right)= \\
\left(p_{1}-p_{2} s\right) q_{r+1} c+p_{2} t\left(b+g_{2} f c\right)=\left(p_{1}-p_{2} s\right)\left(h_{1} a+h_{2} b\right)+p_{2} t\left(b+g_{2} f c\right),
\end{gathered}
$$

pois $q_{r+1} c=h_{1} a+h_{2} b$.

Seja $\eta=\left(p_{1}-p_{2} s\right) h_{1} g_{1} f c=-\left(p_{1}-p_{2} s\right) h_{2} g_{2} f c$. Somando e subtraindo $\eta$ temos:

$$
\begin{gathered}
q_{r} c=\left(p_{1}-p_{2} s\right) h_{1} a+\eta+\left(p_{1}-p_{2} s\right) h_{2} b-\eta+p_{2} t\left(b+g_{2} f c\right)= \\
\left(p_{1}-p_{2} s\right) h_{1} a+\left(p_{1}-p_{2} s\right) h_{1} g_{1} f c+\left(p_{1}-p_{2} s\right) h_{2} b+\left(p_{1}-p_{2} s\right) h_{2} g_{2} f c+p_{2} t\left(b+g_{2} f c\right) .
\end{gathered}
$$

Finalmente,

$$
q_{r} c=\left(p_{1}-p_{2} s\right) h_{1}\left(a+g_{1} f c\right)+\left(\left(p_{1}-p_{2} s\right) h_{2}+p_{2} t\right)\left(b+g_{2} f c\right) .
$$

Tomando $d_{r}=g_{1} f$ e $e_{r}=g_{2} f$, temos que $q_{r} c \in D_{n}\left(a+d_{r} c\right)+D_{n}\left(b+e_{r} c\right)$. 
Agora podemos demonstrar nosso primeiro resultado principal:

Teorema 4.8. Todo ideal à esquerda de $E_{n}(K)=K\left(\left(x_{1}, \ldots, x_{n}\right)\right)\left\langle\partial_{1}, \ldots, \partial_{n}\right\rangle$ pode ser gerado por dois elementos.

Prova: Como o anel $E_{n}(K)=K\left(\left(x_{1}, \ldots, x_{n}\right)\right)\left\langle\partial_{1}, \ldots, \partial_{n}\right\rangle$ é um anel Noetheriano, é suficiente provar que dados $a, b, c \in E_{n}$ existem $d, e \in E_{n}$ tais que $c \in E_{n}(a+d c)+E_{n}(b+e c)$.

Como $a, b, c \in E_{n}$, podemos escrever como somas finitas

$$
a=\sum_{\alpha} \frac{p_{\alpha}}{q} \partial^{\alpha}, b=\sum_{\beta} \frac{p_{\beta}}{q} \partial^{\beta}, c=\sum_{\gamma} \frac{p_{\gamma}}{q} \partial^{\gamma}, \text { onde } p_{\alpha}, p_{\beta}, p_{\gamma}, q \in K\left[\left[x_{1}, \ldots, x_{n}\right]\right], q \neq 0 \text {. }
$$

Sejam $a^{\prime}=q a, b^{\prime}=q b, c^{\prime}=q c \in D_{n}$. Logo, para $r=0$ na proposição anterior, existem $q_{0} \in K\left[\left[x_{1}, \ldots, x_{n}\right]\right], q_{0} \neq 0$ e d $d^{\prime}, e^{\prime} \in D_{n}$ tais que

$q_{0} c^{\prime} \in D_{n}\left(a^{\prime}+d^{\prime} c^{\prime}\right)+D_{n}\left(b^{\prime}+e^{\prime} c^{\prime}\right)$, isto é, $\left(q_{0} q\right) c \in D_{n}\left(q a+d^{\prime} q c\right)+D_{n}\left(q b+e^{\prime} q c\right)$.

Então existem $u^{\prime}, v^{\prime} \in D_{n}$ tais que

$\left(q_{0} q\right) c=u^{\prime}\left(q a+d^{\prime} q c\right)+v^{\prime}\left(q b+e^{\prime} q c\right)=u^{\prime} q(a+d c)+v^{\prime} q(b+e c)$, onde $d=q^{-1} d^{\prime} q, e=q^{-1} e^{\prime} q \in E_{n}$.

Logo

$$
c=u(a+d c)+v(b+e c) \text {, onde } u=\left(q_{0} q\right)^{-1} u^{\prime}, v=\left(q_{0} q\right)^{-1} v^{\prime} \in E_{n} .
$$

Em outras palavras $c \in E_{n}(a+d c)+E_{n}(b+e c)$, para alguns $d, e \in E_{n}$.

OBS: O mesmo é válido para ideais a direita de $E_{n}(K)=K\left(\left(x_{1}, \ldots, x_{n}\right)\right)\left\langle\partial_{1}, \ldots, \partial_{n}\right\rangle$.

\subsection{Os anéis $R_{r}(K)$}

Para cada $0 \leq r \leq n-1$, denotemos com $C_{r}$ o anel de frações do anel $B_{r}(K)=K\left[\left[x_{1}, \ldots, x_{n}\right]\right]\left\langle\partial_{1}, \ldots, \partial_{r}\right\rangle$. Tal anel de frações existe pois $B_{r}(K)$ é um domínio de Ore. Mais ainda $C_{r}(K)$ está contido em $F_{r}\left(\left(x_{r+1}, \ldots, x_{n}\right)\right)$ o anel de frações de $D_{r}\left[\left[x_{r+1}, \ldots, x_{n}\right]\right]$ pois $B_{r}(K) \subset D_{r}\left[\left[x_{r+1}, \ldots, x_{n}\right]\right]$. Ambos $C_{r}(K)$ e $F_{r}\left(\left(x_{r+1}, \ldots, x_{n}\right)\right)$ são anéis de característica zero.

Cada elemento a do anel $F_{r}\left(\left(x_{r+1}, \ldots, x_{n}\right)\right)\left\langle\partial_{r+1}, \ldots, \partial_{n}\right\rangle$ pode ser escrito como uma soma finita $a=\sum_{\alpha} p_{\alpha}\left(x_{r+1}, \ldots, x_{n}\right) \partial^{\alpha}$, onde $p_{\alpha}\left(x_{r+1}, \ldots, x_{n}\right) \in F_{r}\left(\left(x_{r+1}, \ldots, x_{n}\right)\right) e$ $\partial^{\alpha}=\partial_{r+1}^{\alpha_{r+1}} \cdots \partial_{n}^{\alpha_{n}}$. Agora consideremos o seguinte conjunto

$$
R_{r}(K):=\left\{a \in F_{r}\left(\left(x_{r+1}, \ldots, x_{n}\right)\right)\left\langle\partial_{r+1}, \ldots, \partial_{n}\right\rangle, p_{\alpha}\left(x_{r+1}, \ldots, x_{n}\right) \in C_{r}(K) \text { para todo } \alpha\right\}
$$


Não é difícil ver que $R_{r}(K)$ é um subanel de $F_{r}\left(\left(x_{r+1}, \ldots, x_{n}\right)\right)\left\langle\partial_{r+1}, \ldots, \partial_{n}\right\rangle$. Notemos que $D_{n}=K\left[\left[x_{1}, \ldots, x_{n}\right]\right]\left\langle\partial_{1}, \ldots, \partial_{n}\right\rangle$ está contido em $R_{r}(K)$ e que por definição $R_{0}(K)=E_{n}(K)=$ $K\left(\left(x_{1}, \ldots, x_{n}\right)\right)\left\langle\partial_{1}, \ldots, \partial_{n}\right\rangle$.

Nas seguintes proposições, para simplificar a notação, escreveremos $R_{r}, B_{r}$ e $C_{r}$ no lugar de $R_{r}(K), B_{r}(K)$ e $C_{r}(K)$, respectivamente.

Proposição 4.9. Seja $r$ um inteiro não negativo tal que $0 \leq r \leq n-1$ e seja q um elemento diferente de zero de $B_{r+1}=K\left[\left[x_{1}, \ldots, x_{n}\right]\right]\left\langle\partial_{1}, \ldots, \partial_{r+1}\right\rangle$ e sejam u e v elementos de $D_{n}$ tal que $v \neq 0$. Então existe algum $f$ em $D_{n}$ tal que

$$
R_{r}=R_{r} q+R_{r}(u+v f) .
$$

Prova: Fixemos $r$ tal que $0 \leq r \leq n-1$. Desde que $v \neq 0$, podemos escrever

$$
v=\delta_{1} G_{1}+\cdots+\delta_{m} G_{m}
$$

onde $\delta_{1}, \ldots, \delta_{m}$ são elementos de $K\left[\left[x_{1}, \ldots, x_{n}\right]\right]\left\langle\partial_{r+1}\right\rangle$ e $G_{1}, \ldots, G_{m}$ são elementos de

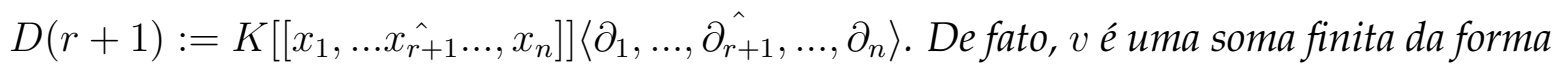
$v=\sum p_{\alpha} \partial_{1}^{\alpha_{1}} \cdots \partial_{r+1}^{\alpha_{r+1}} \cdots \partial_{n}^{\alpha_{n}}$, o que pode ser escrita como $v=\sum\left(p_{\alpha} \partial_{r+1}^{\alpha_{r+1}}\right)\left(\partial_{1}^{\alpha_{1}} \cdots \partial_{r+1}^{\hat{\alpha}_{r+1}} \cdots \partial_{n}^{\alpha_{n}}\right)$ $\operatorname{com} p_{\alpha} \partial_{r+1}^{\alpha_{r+1}} \in K\left[\left[x_{1}, \ldots, x_{n}\right]\right]\left\langle\partial_{r+1}\right\rangle$ e $\partial_{1}^{\alpha_{1}} \cdots \partial_{r+1}^{\alpha_{r+1}} \cdots \partial_{n}^{\alpha_{n}} \in D(r+1)$.

Agora observemos que $K\left[\left[x_{1}, \ldots, x_{n}\right]\right]=\left(K\left[\left[x_{1}, \ldots x_{r+1}, \ldots, x_{n}\right]\right]\right)\left[\left[x_{r+1}\right]\right]$ está contido em $F\left[\left[x_{r+1}\right]\right]$, onde $F$ é o corpo de frações de $K\left[\left[x_{1}, \ldots, x_{r+1}, \ldots, x_{n}\right]\right]$. Logo, a característica de $F$ é zero e $\delta_{i}$ é um elemento de $F\left[\left[x_{r+1}\right]\right]\left\langle\partial_{r+1}\right\rangle$, para todo $i=1, \ldots, m$.

Seja $T$ o anel de frações do domínio de Ore $K\left[\left[x_{1}, \ldots, x_{r+1}, \ldots, x_{n}\right]\right]\left\langle\partial_{1}, \ldots, \partial_{r}\right\rangle=$ $\left\{q \in D_{r}\left[\left[x_{r+2}, \ldots, x_{n}\right]\right]\right.$, q tém ordem finita em $x_{i}$ para todo $\left.1 \leq i \leq r\right\}$. Então $T$ é um anel de divisão e F está contido em $T$.

Desde que $v$ é diferente de zero, temos que $G_{i}$ é diferente de zero 0 para algum $1 \leq i \leq m$. Como $D(r+1)$ é um anel simples, então o ideal bilateral gerado por $G_{1}, \ldots, G_{m}$ é o todo o anel $D(r+1)$. Por tanto existem elementos $a_{1}, \ldots, a_{l}$ e $b_{1}, \ldots, b_{l}$ em $D(r+1)$ tal que $1=$ $\sum_{j=1}^{m} \sum_{\nu=1}^{l} b_{\nu} G_{j} a_{\nu}$ e por tanto $D(r+1)=\Sigma \Sigma D(r+1) G_{j} a_{\nu}$. Identificando $D(r+1)$ com um subanel de $R_{r}$ concluimos que $R_{r}=\Sigma \Sigma R_{r} G_{j} a_{\nu}$.

Neste momento precisamos da seguinte

Afirmação: Para cada m-upla $B_{1}, \ldots, B_{m}$ em $D(r+1)$ existe algum $f \in Z\left[\left[x_{r+1}\right]\right]\left\langle\partial_{r+1}\right\rangle$ tal que

$$
R_{r} q+R_{r} u+R_{r} B_{1}+\cdots+R_{r} B_{m}=R_{r} q+R_{r}\left(u+\delta_{1} f B_{1}+\cdots+\delta_{m} f B_{m}\right) .
$$

Com efeito, desde que q é um elemento diferente de zero em $B_{r+1}$, segue-se da proposição 4.3 
que existe algum $\rho$ diferente de zero em $B_{r+1}$ tal que $\rho B_{j} \in D_{n} q$ para todo $j=1, \ldots$, m e também $\rho u \in D_{n} q$.

Seja $S=T\left(\left(x_{r+1}\right)\right)\left\langle\partial_{r+1}\right\rangle$, então $0 \neq \rho \in S$. Usando a proposição $3.10(i)$, temos que existe algum $f \in \mathbb{Z}\left[\left[x_{r+1}\right]\right]\left\langle\partial_{r+1}\right\rangle$ tal que $S^{(m+1)}=S^{(m+1)} \rho+S\left(\varepsilon_{0}+\delta_{1} f \varepsilon_{1}+\cdots+\delta_{1} f \varepsilon_{m}\right)$. Desde $S$ é um subanel de $R_{r}$, temos que $R_{r}^{(m+1)}=R_{r}^{(m+1)} \rho+R_{r}\left(\varepsilon_{0}+\delta_{1} f \varepsilon_{1}+\cdots+\delta_{1} f \varepsilon_{m}\right)$.

Consideremos a $R_{r}$ - aplicação linear $\pi: R_{r}^{(m+1)} \rightarrow R_{r}$ definida por $\pi\left(\varepsilon_{0}\right)=u$ e $\pi\left(\varepsilon_{j}\right)=B_{j}$ para cada $1 \leq j \leq m$. Então a imagem de $\pi$ é $R_{r} u+R_{r} B_{1}+\ldots+R_{r} B_{m} \subseteq R_{r}$; mas $\rho u$, $\rho B_{j} \in D_{n} q$. Então temos que $\pi\left(\rho \varepsilon_{0}\right)=\rho \pi\left(\varepsilon_{0}\right)=\rho u \in R_{r} q$ e $\pi\left(\rho \varepsilon_{j}\right)=\rho \pi\left(\varepsilon_{j}\right)=\rho B_{j} \in R_{r} q$ para cada $1 \leq j \leq m$. Por tanto $\pi\left(R_{r}^{(m+1)} \rho\right) \subseteq R_{r} q$. Então

$$
\begin{gathered}
R_{r} q+R_{r} u+R_{r} B_{1}+\cdots+R_{r} B_{m}=R_{r} q+\pi\left(R_{r}^{(m+1)}\right) \\
\subseteq R_{r} q+\pi\left(R_{r}^{(m+1)} \rho+R_{r}\left(\varepsilon_{0}+\delta_{1} f \varepsilon_{1}+\cdots+\varepsilon_{0}+\delta_{m} f \varepsilon_{m}\right)\right) \\
\subseteq R_{r} q+\pi\left(R_{r}^{(m+1)} \rho\right)+R_{r}\left(u+\delta_{1} f B_{1}+\cdots+\delta_{m} f B_{m}\right) \\
\subseteq R_{r} q+R_{r}\left(u+\delta_{1} f B_{1}+\cdots+\delta_{m} f B_{m}\right)
\end{gathered}
$$

e isto prova a afirmação pois a inclusão oposta é clara.

Se agora aplicamos a afirmação a $B_{j}=G_{j} a_{1}, j=1, \ldots, m$. Então, existe algum $f_{1} \in$ $\mathbb{Z}\left[\left[x_{r+1}\right]\right]\left\langle\partial_{r+1}\right\rangle$ tal que

$$
R_{r} q+R_{r} u+R_{r} G_{1} a_{1}+\cdots+R_{r} G_{m} a_{1}=R_{r} q+R_{r}\left(u+\delta_{1} f_{1} G_{1} a_{1}+\cdots+\delta_{m} f_{1} G_{m} a_{1}\right) .
$$

Desde que $f_{1} \in \mathbb{Z}\left[\left[x_{r+1}\right]\right]\left\langle\partial_{r+1}\right\rangle$ e $G_{j} \in D(r+1)$ comutam, temos que $\delta_{1} f_{1} G_{1} a_{1}+\cdots+\delta_{m} f_{1} G_{m} a_{1}=\delta_{1} G_{1} f_{1} a_{1}+\cdots+\delta_{m} G_{m} f_{1} a_{1}=\left(\delta_{1} G_{1} a_{1}+\cdots+\delta_{m} G_{m}\right) f_{1} a_{1}=v f_{1} a_{1}$, pois $v=\delta_{1} G_{1}+\cdots+\delta_{m} G_{m}$. Por tanto,

$$
R_{r} q+R_{r} u+R_{r} G_{1} a_{1}+\cdots+R_{r} G_{m} a_{1}=R_{r} q+R_{r}\left(u+v f_{1} a_{1}\right) .
$$

Agora aplicamos a afirmação, mas substituímos $u$ por $u+v f_{1} a_{1}$ and $B_{j}=G_{j} a_{2}, j=$ $1, \ldots, m$. Então existe $f_{2} \in \mathbb{Z}\left[\left[x_{r+1}\right]\right]\left\langle\partial_{r+1}\right\rangle$ tal que $R_{r} q+R_{r}\left(u+v f_{1} a_{1}\right)+\sum R_{r} G_{j} a_{2}=$ $R_{r} q+R_{r}\left(u+v f_{1} a_{1}+v f_{2} a_{2}\right)$. Usando a equação previa temos que

$$
R_{r} q+R_{r} u+\sum R_{r} G_{j} a_{1}+\sum R_{r} G_{j} a_{2}=R_{r} q+R_{r}\left(u+v f_{1} a_{1}+v f_{2} a_{2}\right) .
$$

No seguinte passo aplicamos a afirmação com $B_{j}=G_{j} a_{3}, j=1, \ldots$, m e u substituído por 
$u+v f_{1} a_{1}+v f_{2} a_{2}$. Depois de l passos temos que

$$
R_{r} q+R_{r}\left(u+v f_{1} a_{1}+\cdots+v f_{l} a_{l}\right)=R_{r} q+R_{r} u+\sum \sum R_{r} G_{j} a_{\nu}=R_{r} .
$$

Por tanto a proposição segue-se com $f=f_{1} a_{1}+\cdots+f_{l} a_{l}$.

Agora podemos demonstrar a Proposição 4.6.

\section{Prova da Proposição 4.6}

\section{Prova:}

A proposição 4.9 implica a existência de algum $f \in D_{n}$ tal que $1=s q+t(u+v f)$ para alguns elementos $s, t \in R_{r}$. Então $s, t$ podem ser escritos como uma soma finita $s=\sum_{\alpha} p_{\alpha}\left(x_{r+1}, \ldots, x_{n}\right) \partial^{\alpha}, t=\sum_{\beta} p_{\beta}\left(x_{r+1}, \ldots, x_{n}\right) \partial^{\beta}$, onde cada $p_{\alpha}\left(x_{r+1}, \ldots, x_{n}\right)$ e cada $p_{\beta}\left(x_{r+1}, \ldots, x_{n}\right)$ são elementos de $C_{r}$, o anel de frações de $B_{r}$. Então $p_{\alpha}=b_{\alpha}^{-1} a_{\alpha} e$ $p_{\beta}=b_{\beta}^{-1} a_{\beta}$, para alguns $a_{\alpha}, b_{\alpha}, a_{\beta}, b_{\beta} \in B_{r}, b_{\alpha} \neq 0, b_{\beta} \neq 0$. Logo $s=\sum_{\alpha} b_{\alpha}^{-1} a_{\alpha} \partial^{\alpha}$ e $t=\sum_{\beta} b_{\beta}^{-1} a_{\beta} \partial^{\beta}$. Desde que $B_{r}$ é um domínio de Ore, existe um múltiplo comum $q_{r} \in B_{r}$ dos $b_{\alpha}$ e dos $b_{\beta}$, ou seja, para todo $\alpha, \beta$ temos que $q_{r} \in B_{r} a_{\beta}$ e $q_{r} \in B_{r} b_{\beta}$. Logo $s=\sum_{\alpha} q_{r}^{-1} c_{\alpha} \partial^{\alpha}=q_{r}^{-1}\left(\sum_{\alpha} c_{\alpha} \partial^{\alpha}\right) e t=\sum_{\beta} q_{r}^{-1} c_{\beta} \partial^{\beta}=q_{r}^{-1}\left(\sum_{\beta} c_{\beta} \partial^{\beta}\right)$, para alguns $c_{\alpha}, c_{\beta} \in B_{r}$. Portanto $s=q^{-1} s_{1}$ e $t=q^{-1} t_{1}$, onde $s_{1}:=\sum_{\alpha} c_{\alpha} \partial^{\alpha} \in D_{n}$ e $t_{1}:=\sum_{\beta} c_{\beta} \partial^{\beta} \in$ $D_{n}$. Logo $1=q_{r}^{-1} s_{1} q+q_{r}^{-1} t_{1}(u+v f)$. Em consequência temos que $q_{r}=s_{1} q+t_{1}(u+v f) \in$ $D_{n} q+D_{n}(u+v f)$.

\subsection{Os anéis $S_{r}(K)$}

Seja $K$ um corpo de característica zero e seja $r$ um inteiro não negativo tal que $0 \leq r \leq n$. Introduzimos o anel $S_{r}(K)=K\left(\left(x_{1}, \ldots, x_{r}\right)\right)\left[\left[x_{r+1}, \ldots, x_{n}\right]\right]\left\langle\partial_{1}, \ldots, \partial_{n}\right\rangle$, que é um quociente parcial de $D_{n}(K)=K\left[\left[x_{1}, \ldots, x_{n}\right]\right]\left\langle\partial_{1}, \ldots \partial_{n}\right\rangle$. Se $v \in S_{r}$, então $v=q^{-1} a$, para algum $a \in$ $D_{n}(K)$ e algum $q \in K\left[\left[x_{1}, \ldots, x_{r}\right]\right]$. Por definição $S_{0}(K)=D_{n}(K)$ e $S_{n}(K)=E_{n}(K)=$ $K\left(\left(x_{1}, \ldots, x_{n}\right)\right)\left\langle\partial_{1}, \ldots, \partial_{n}\right\rangle$. Quando o corpo $K$ está fixado, usaremos a notação $S_{r}$ no lugar de $S_{r}(K)$.

Proposição 4.10. Seja $0 \leq r \leq n-1$ e seja $q \in K\left[\left[x_{1}, \ldots, x_{r+1}\right]\right], q \neq 0$. Se $a_{1}, \ldots, a_{t}$ são elementos de $D_{n}(K)$. Então existe algum $\rho \in K\left[\left[x_{1}, \ldots, x_{n}\right]\right], \rho \neq 0$ tal que $\rho a_{j} \in D_{n}$ q para todo $1 \leq j \leq t$.

Prova: Cada $a_{j}$ pode ser escrito como uma soma finita $\sum_{\alpha} p_{j \alpha} \partial^{\alpha}$, onde $p_{j \alpha} \in K\left[\left[x_{1}, \ldots, x_{n}\right]\right]$ e $\partial^{\alpha}=\partial_{1}^{\alpha_{1}} \ldots \partial_{n}^{\alpha_{n}}$. Escolhemos um inteiro positivo $w$ suficientemente grande tal que $p_{j \alpha}=0$, para todo $|\alpha| \geq w$.

Como $K\left[\left[x_{1}, \ldots, x_{n}\right]\right]$ é um domínio de Ore e $q^{w} \in K\left[\left[x_{1}, \ldots, x_{n}\right]\right], q^{w} \neq 0$, então existe algum $\rho \in K\left[\left[x_{1}, \ldots, x_{n}\right]\right], \rho \neq 0$ tal que $\rho p_{j \alpha} \in K\left[\left[x_{1}, \ldots, x_{n}\right]\right] q^{w}$, para todo $j$ e $\alpha$. 
$\operatorname{Logo} \rho p_{j \alpha}=s_{j \alpha} q^{w}$ para algum $s_{j \alpha} \in K\left[\left[x_{1}, \ldots, x_{n}\right]\right]$. Então temos que

$$
\rho a_{j}=\sum_{|\alpha|<w}\left(\rho p_{j \alpha}\right) \partial^{\alpha}=\sum_{|\alpha|<w}\left(s_{j \alpha} q^{w}\right) \partial^{\alpha}=\sum_{|\alpha|<w} s_{j \alpha}\left(q^{w} \partial^{\alpha}\right)
$$

Por outro lado, $q^{w} \partial^{\alpha} \in D_{n} q$ se $|\alpha|<w$. De fato, $q^{w} \partial_{1}^{\alpha_{1}}=\lambda_{1} q^{w-\alpha_{1}}$, para algum $\lambda_{1} \in D_{n}$. Logo $q^{w} \partial_{1}^{\alpha_{1}} \partial_{2}^{\alpha_{2}}=\lambda_{1}\left(q^{w-\alpha_{1}} \partial_{2}^{\alpha_{2}}\right)=\lambda_{1} \lambda_{2} q^{w-\left(\alpha_{1}+\alpha_{2}\right)}$, para algum $\lambda_{2} \in D_{n}$. Continuando assim temos que $q^{w} \partial_{1}^{\alpha_{1}} \cdots \partial_{n}^{\alpha_{n}}=\left(\lambda_{1} \cdots \lambda_{n}\right) q^{w-\left(\alpha_{1}+\cdots+\alpha_{n}\right)}$ e como $|\alpha|<w$, temos que $q^{w} \partial^{\alpha} \in D_{n} q$. Isto completa a prova.

Agora provaremos que todo ideal à esquerda do anel $S_{n-1}=K\left(\left(x_{1}, \ldots, x_{n-1}\right)\right)\left[\left[x_{n}\right]\right]\left\langle\partial_{1}, \ldots, \partial_{n}\right\rangle$ é 2-gerado. Isso será consequência da seguinte proposição:

Proposição 4.11. Sejam $a, b$ e c elementos diferentes de zero de $D_{n}$. Então existem $q_{1} \in$ $K\left[\left[x_{1}, \ldots, x_{n-1}\right]\right], q_{1} \neq 0, d, e \in D_{n}$ tais que

$$
q_{1} c \in D_{n}(a+d c)+D_{n}(b+e c)
$$

Para demonstrar a proposição acima precisamos do seguinte resultado, cuja prova será adiada até aparte final da seção.

Proposição 4.12. Seja $q \in K\left[\left[x_{1}, \ldots, x_{n}\right]\right], q \neq 0$ e sejam $u, v \in D_{n}$ tais que $v \neq 0$. Então existem $q_{1} \in K\left[\left[x_{1}, \ldots, x_{n-1}\right]\right]$ e $f \in D_{n}$ tais que

$$
q_{1} \in D_{n} q+D_{n}(u+v f) .
$$

\section{Prova da Proposição 4.11}

Prova: Pela Proposição 4.5, para $r=0$, existem $q_{0} \in B_{0}(K)=K\left[\left[x_{1}, \ldots, x_{n}\right]\right], q_{0} \neq 0 e$ $d_{0}, e_{0} \in D_{n}$ tais que

$$
q_{0} c \in D_{n}\left(a+d_{0} c\right)+D_{n}\left(b+e_{0} c\right) .
$$

Se repetirmos o argumento da prova da proposição 4.5 e fazemos uso da proposição 4.12 no lugar da proposição 4.6 , obtemos $q_{1} \in K\left[\left[x_{1}, \ldots, x_{n-1}\right]\right]$ e $d, e \in D_{n}$ tais que

$$
q_{1} c \in D_{n}(a+d c)+D_{n}(b+e c)
$$

Agora podemos demonstrar nosso segundo resultado principal : 
Teorema 4.13. Todo ideal à esquerda de $S_{n-1}=K\left(\left(x_{1}, \ldots, x_{n-1}\right)\right)\left[\left[x_{n}\right]\right]\left\langle\partial_{1}, \ldots, \partial_{n}\right\rangle$ pode ser gerado por dois elementos.

Prova: Como o anel $S_{n-1}=K\left(\left(x_{1}, \ldots, x_{n-1}\right)\right)\left[\left[x_{n}\right]\right]\left\langle\partial_{1}, \ldots, \partial_{n}\right\rangle$ é Noetheriano, é suficiente provar que dados $a, b, c \in S_{n-1}$ distintos de zero, existem $d, e \in S_{n-1}$ tais que

$$
c \in S_{n-1}(a+d c)+S_{n-1}(b+e c) .
$$

Como $a, b, c \in S_{n-1}$, existe $q^{\prime} \in K\left[\left[x_{1}, \ldots, x_{n-1}\right]\right]$ tais que $a^{\prime}=q^{\prime} a, b^{\prime}=q^{\prime} b, c^{\prime}=q^{\prime} c \in D_{n}$ e $a^{\prime} b^{\prime} c^{\prime} \neq 0$. Logo, pela proposição 4.11, existem $q_{1} \in K\left[\left[x_{1}, \ldots, x_{n-1}\right]\right], q_{1} \neq 0, d_{1}, e_{1} \in D_{n}$ tais que

$$
q_{1} c^{\prime} \in D_{n}\left(a^{\prime}+d_{1} c^{\prime}\right)+D_{n}\left(b^{\prime}+e_{1} c^{\prime}\right) .
$$

Logo, como no teorema 4.8 .

$$
c \in S_{n-1}(a+d c)+S_{n-1}(b+e c),
$$

para alguns $d, c \in D_{n}$.

Corolário 4.14. Todo ideal à esquerda do anel $D_{1}(K)=K[[x]]\left\langle\partial_{x}\right\rangle$ pode ser gerado por dois elementos.

Prova: Tome $n=1$ no Teorema anterior e observe que $S_{0}(K)=K\left[\left[x_{1}\right]\right]\left\langle\partial_{1}\right\rangle=D_{1}(K)$.

Antes de demonstrar a Proposição 4.12, mostraremos o seguinte:

Proposição 4.15. Seja $q \in K\left[\left[x_{1}, \ldots, x_{n-1}\right], q \neq 0\right.$ e sejam $u, v \in D_{n}$ tal que $v \neq 0$. Então existe algum $f \in D_{n}$ tal que $S_{n-1}=S_{n-1} q+S_{n-1}(u+v f)$.

Prova: Desde que $0 \neq v \in D_{n}$, podemos escrever

$$
v=\delta_{1} G_{1}+\cdots+\delta_{m} G_{m}
$$

onde $\delta_{1}, \ldots, \delta_{m} \in K\left[\left[x_{1}, . ., x_{n}\right]\right]\left\langle\partial_{n}\right\rangle e G_{1}, \ldots, G_{m} \in K\left[\left[x_{1}, \ldots, x_{n-1}\right]\right]\left\langle\partial_{1}, \ldots, \partial_{n-1}\right\rangle$.

Se $F:=K\left(\left(x_{1}, \ldots, x_{n-1}\right)\right)$ é o corpo de frações de $K\left[\left[x_{1}, \ldots, x_{n-1}\right]\right]$, então $F$ é um corpo de característica zero e $\delta_{i} \in F\left[\left[x_{n}\right]\right]\left\langle\partial_{n}\right\rangle$ para todo $i=1, \ldots, m$.

Desde que $v \neq 0$, temos que $G_{i} \neq 0$, para algum $i=1, \ldots, m$. Como o anel $D_{n-1}(K)=$ $K\left[\left[x_{1}, \ldots, x_{n-1}\right]\right]\left\langle\partial_{1}, \ldots, \partial_{n-1}\right\rangle$ é simples, então o ideal bilateral gerado por $G_{1}, \ldots, G_{m}$ é todo $o$ anel $D_{n-1}(K)$. Portanto existem elementos $a_{1}, \ldots, a_{l}$ e $b_{1}, \ldots, b_{l}$ in $D_{n-1}(K)$ tais que $1=$ 
$\sum_{j=1}^{m} \sum_{\nu=1}^{l} b_{\nu} G_{j} a_{\nu}$ e portanto $D_{n-1}(K)=\Sigma \Sigma K\left[\left[x_{1}, \ldots, x_{n-1}\right]\right] D_{n-1}(K) G_{j} a_{\nu}$. Identificando $D_{n-1}(K)$ com um subanel de $S_{n-1}=K\left(\left(x_{1}, \ldots, x_{n-1}\right)\right)\left[\left[x_{n}\right]\right]\left\langle\partial_{1}, \ldots, \partial_{n}\right\rangle$ concluimos que $S_{n-1}=\Sigma \Sigma S_{n-1} G_{j} a_{\nu}$.

Neste momento precisamos da seguinte

Afirmação: Para cada m-upla $B_{1}, \ldots, B_{m}$ em $D_{n-1}(K)$ existe algum $f \in Z\left[\left[x_{n}\right]\right]\left\langle\partial_{n}\right\rangle$ tal que

$$
S_{n-1} q+S_{n-1} u+S_{n-1} B_{1}+\cdots+S_{n-1} B_{m}=S_{n-1} q+S_{n-1}\left(u+\delta_{1} f B_{1}+\cdots+\delta_{m} f B_{m}\right) .
$$

Com efeito, desde que q é um elemento diferente de zero de $K\left[\left[x_{1}, \ldots, x_{n-1}\right]\right]$, segue-se da proposição 4.10 que existe algum $\rho \in K\left[\left[x_{1}, \ldots, x_{n}\right]\right], \rho \neq 0$, tal que $\rho B_{j} \in D_{n}$ q para todo $j=1, \ldots, m$ e também $\rho u \in D_{n} q$

Seja $D=F\left[\left[x_{n}\right]\right]\left\langle\partial_{n}\right\rangle=K\left(\left(x_{1}, \ldots, x_{n-1}\right)\right)\left[\left[x_{n}\right]\right]\left\langle\partial_{n}\right\rangle$, então $0 \neq \rho \in D$. Usando a proposição $3.10($ ii $)$ temos que existe algum $f \in \mathbb{Z}\left[\left[x_{n}\right]\right]\left\langle\partial_{n}\right\rangle$ tal que

$$
D^{m+1}=D^{m+1} \rho+D\left(\varepsilon_{0}+\delta_{1} f \varepsilon_{1}+\cdots+\delta_{1} f \varepsilon_{m}\right) .
$$

Desde que Dé um subanel de $S_{n-1}$, temos que

$$
S_{n-1}^{m+1}=S_{n-1}^{m+1} \rho+S_{n-1}\left(\varepsilon_{0}+\delta_{1} f \varepsilon_{1}+\cdots+\delta_{1} f \varepsilon_{m}\right)
$$

Consideremos a $S_{n-1}$ - aplicação linear $\pi: S_{n-1}^{(m+1)} \rightarrow S_{n-1}$ definida por $\pi\left(\varepsilon_{0}\right)=u e$ $\pi\left(\varepsilon_{j}\right)=B_{j}$ para cada $1 \leq j \leq m$. Então a imagem de $\pi$ é $S_{n-1} u+S_{n-1} B_{1}+\ldots+S_{n-1} B_{m} \subseteq$ $S_{n-1} ;$ mas $\rho u, \rho B_{j} \in D_{n} q$. Então temos que $\pi\left(\rho \varepsilon_{0}\right)=\rho \pi\left(\varepsilon_{0}\right)=\rho u \in S_{n-1} q$ e $\pi\left(\rho \varepsilon_{j}\right)=$ $\rho \pi\left(\varepsilon_{j}\right)=\rho B_{j} \in S_{n-1}$ q para cada $1 \leq j \leq m$. Portanto $\pi\left(R_{r}^{(m+1)} \rho\right) \subseteq S_{n-1} q$. Logo

$$
\begin{gathered}
S_{n-1} q+S_{n-1} u+S_{n-1} B_{1}+\cdots+S_{n-1} B_{m}=S_{n-1} q+\pi\left(S_{n-1}^{(m+1)}\right) \\
\subseteq S_{n-1} q+\pi\left(S_{n-1}^{(m+1)} \rho+S_{n-1}\left(\varepsilon_{0}+\delta_{1} f \varepsilon_{1}+\cdots+\varepsilon_{0}+\delta_{m} f \varepsilon_{m}\right)\right) \\
\subseteq S_{n-1} q+\pi\left(S_{n-1}^{(m+1)} \rho\right)+S_{n-1}\left(u+\delta_{1} f B_{1}+\cdots+\delta_{m} f B_{m}\right) \\
\subseteq S_{n-1} q+S_{n-1}\left(u+\delta_{1} f B_{1}+\cdots+\delta_{m} f B_{m}\right)
\end{gathered}
$$

e isto prova a afirmação pois a inclusão oposta é clara.

Se agora aplicamos a afirmação a $B_{j}=G_{j} a_{1}, j=1, \ldots, m$. Então, existe algum $f_{1} \in$ $\mathbb{Z}\left[\left[x_{n}\right]\right]\left\langle\partial_{n}\right\rangle$ tal que

$S_{n-1} q+S_{n-1} u+S_{n-1} G_{1} a_{1}+\cdots+S_{1} G_{m} a_{1}=S_{n-1} q+S_{n-1}\left(u+\delta_{1} f_{1} G_{1} a_{1}+\cdots+\delta_{m} f_{1} G_{m} a_{1}\right)$. 
Desde que $f_{1} \in \mathbb{Z}\left[\left[x_{n}\right]\right]\left\langle\partial_{n}\right\rangle$ e $G_{j} \in D_{n-1}$ comutam, temos que

$\delta_{1} f_{1} G_{1} a_{1}+\cdots+\delta_{m} f_{1} G_{m} a_{1}=\delta_{1} G_{1} f_{1} a_{1}+\cdots+\delta_{m} G_{m} f_{1} a_{1}=\left(\delta_{1} G_{1}+\cdots+\delta_{m} G_{m}\right) f_{1}=v f_{1} a_{1}$,

pois $v=\delta_{1} G_{1}+\cdots+\delta_{m} G_{m}$. Portanto

$$
S_{n-1} q+S_{n-1} u+S_{n-1} G_{1} a_{1}+\cdots+S_{1} G_{m} a_{1}=S_{1} q+S_{1}\left(u+v f_{1} a_{1}\right) .
$$

Agora aplicamos a afirmação, mas substituímos u por $u+v f_{1} a_{1}$ e $B_{j}=G_{j} a_{2}, j=1, \ldots, m$. Então existe $f_{2} \in \mathbb{Z}\left[\left[x_{n}\right]\right]\left\langle\partial_{n}\right\rangle$ tal que

$$
S_{n-1} q+S_{n-1}\left(u+v f_{1} a_{1}\right)+\sum S_{n-1} G_{j} a_{2}=S_{n-1} q+S_{n-1}\left(u+v f_{1} a_{1}+v f_{2} a_{2}\right) .
$$

Usando a equação previa temos que

$$
S_{n-1} q+S_{n-1} u+\sum S_{n-1} G_{j} a_{1}+\sum S_{n-1} G_{j} a_{2}=S_{n-1} q+S_{n-1}\left(u+v f_{1} a_{1}+v f_{2} a_{2}\right) .
$$

No seguinte passo aplicamos a afirmação com $B_{j}=G_{j} a_{3}, j=1, \ldots$, m e u substituído por $u+v f_{1} a_{1}+v f_{2} a_{2}$. Depois de l passos temos que

$$
S_{n-1} q+S_{n-1}\left(u+v f_{1} a_{1}+\cdots+v f_{l} a_{l}\right)=S_{n-1} q+S_{n-1} u+\sum \sum S_{n-1} G_{j} a_{\nu}=S_{n-1} .
$$

Portanto a proposição segue-se com $f=f_{1} a_{1}+\cdots+f_{l} a_{l}$.

Agora provemos a proposição 4.12

\section{Prova da Proposição 4.12}

Prova: A proposição 4.15 implica que existe algum $f \in D_{n}$ tal que

$1=s q+t(u+v f)$, para alguns $s, t \in S_{n_{1}}=K\left(\left(x_{1}, \ldots, x_{n-1}\right)\right)\left[\left[x_{n}\right]\right]\left\langle\partial_{n}\right\rangle$. Como $S_{n-1} e ́$ um quociente parcial de $D_{n}$, existe $q \in K\left[\left[x_{1}, \ldots, x_{n-1}\right]\right], q \neq 0$ tal que $q_{1} s=a_{1} \in D_{n}$ e $q_{1} t=a_{2} \in D_{n}$. Portanto $q_{1}=a_{1} q+a_{2}(u+v f) \in D_{n} q+D_{n}(u+v f)$. 


\section{Referências Bibliográficas}

[1] J.-E. Björk. Rings of differential operators, volume 21 of North-Holland Mathematical Library. North-Holland Publishing Co., Amsterdam, 1979.

[2] P. M. Cohn. Skew fields, volume 57 of Encyclopedia of Mathematics and its Applications. Cambridge University Press, Cambridge, 1995. Theory of general division rings.

[3] S. C. Coutinho. A primer of algebraic D-modules, volume 33 of London Mathematical Society Student Texts. Cambridge University Press, Cambridge, 1995.

[4] S. C. Coutinho and M. P. Holland. Module structure of rings of differential operators. Proc. London Math. Soc. (3), 57(3):417-432, 1988.

[5] K. R. Goodearl and R. B. Warfield, Jr. An introduction to noncommutative Noetherian rings, volume 61 of London Mathematical Society Student Texts. Cambridge University Press, Cambridge, second edition, 2004.

[6] M. Granger and P. Maisonobe. A basic course on differential modules. In Éléments de la théorie des systèmes différentiels. $D$-modules cohérents et holonomes (Nice, 1990), volume 45 of Travaux en Cours, pages 103-168. Hermann, Paris, 1993.

[7] A. Hillebrand and W. Schmale. Towards an effective version of a theorem of Stafford. J. Symbolic Comput., 32(6):699-716, 2001. Effective methods in rings of differential operators.

[8] T. Y. Lam. Lectures on modules and rings, volume 189 of Graduate Texts in Mathematics. Springer-Verlag, New York, 1999.

[9] S. Lang. Algebra, volume 211 of Graduate Texts in Mathematics. Springer-Verlag, New York, third edition, 2002. 
[10] A. Leykin. Algorithmic proofs of two theorems of Stafford. J. Symbolic Comput., 38(6):1535-1550, 2004.

[11] J. C. McConnell and J. C. Robson. Noncommutative Noetherian rings, volume 30 of Graduate Studies in Mathematics. American Mathematical Society, Providence, RI, revised edition, 2001. With the cooperation of L. W. Small.

[12] J. T. Stafford. Module structure of Weyl algebras. J. London Math. Soc. (2), 18(3):429442, 1978. 